\title{
Epigenetic Dysregulation of the Expression of PRSS3 Splice Variants Increases the Heterogeneity of Transcripts and Functionality in Human Hepatocellular Carcinoma
}

Shuye Lin

Beijing Chest Hospital https://orcid.org/0000-0002-4292-0302

Hanli Xu

Beijing Jiaotong University

Mengdi Pang

Beijing Jiaotong University

Xiaomeng Zhou

Beijing Jiaotong University

Yuanming Pan

Beijing Chest Hospital

Lishu Zhang

Beijing Jiaotong University

\section{Xin Guan}

Beijing Jiaotong University

Xiaoyue Wang

Beijing Jiaotong University

Bonan Lin

Beijing Jiaotong University

Rongmeng Tian

Beijing Jiaotong University

Keqiang Chen

National Institutes of Health

Xiaochen Zhang

Beijing Jiaotong University

Zijiang Yang

Beijing Jiaotong University

Fengmin Ji

Beijing Jiaotong University

Yingying Huang

Chinese Academy of Sciences 


\section{Wu Wei}

Chinese Academy of Sciences

\section{Wanghua Gong}

National Institutes of Health

Jianke Ren

Chinese Academy of Sciences

\section{Ji Ming Wang}

National Institutes of Health

\section{Mingzhou Guo}

Chinese PLA General Hospital

Jiaqiang Huang ( $\sim$ huangjiaqiang@bjxkyy.cn )

Beijing Chest Hospital https://orcid.org/0000-0002-6610-8159

\section{Primary research}

Keywords: human hepatocellular carcinoma, PRSS3, splice variants, transcript heterogeneity, CpG methylation, biomarker

Posted Date: September 9th, 2021

DOI: https://doi.org/10.21203/rs.3.rs-861068/v1

License: (c) (i) This work is licensed under a Creative Commons Attribution 4.0 International License. Read Full License 


\section{Abstract \\ Background}

Hepatocellular carcinoma (HCC) is one of the most lethal human tumors with extensive heterogeneity. Serine protease 3 (PRSS3) is an indispensable member of the trypsin family and has been implicated in the pathogenesis of several malignancies including HCC. However, paradoxical effects of PPRSS3 on carcinogenesis impede the utilization of its biomarker potential. We hereby systematically dissected the expression of four known splice variants of PRSS3 (PRSS3-SVS) and their functional relevance to HCC.

\section{Methods}

The expression and DNA methylation of PRSS3 transcripts and their associated clinical relevance in HCC were analyzed using several publicly available datasets and were validated using qPCR-based assays. Functional assays were performed on gain- and loss-of-function cell models, in which PRSS3 transcript constructs were separately transfected after PRSS3 expression was knocked out by CRISPR-Cas 9 editing.

\section{Results}

PRSS3 expression was differentially decreased in HCC cell lines and tissues attributable to aberrant expression of PRSS3-SVs towards bipolarity, in which PRSS3-V2 and then -V1 were dominantly expressed whereas $P R S S 3-V 3$ and $-V 4$ were rarely or minimally expressed. The expression of PRSS3-V2 or $-V 1$ was reversely associated with site-specific CpG methylation and was distinct between lowly-expressed PRSS3$S V s$ with hypermethylation (mPRSS3 ${ }^{L O M}$ ) and highly-expressed PRSS3-SVs with hypomethylation (umPRSS3 ${ }^{H i g h}$ ). Function analysis revealed that PRSS3-V2 exhibited oncogenic functions distinct from a tumor-suppressive role of ectopic PRSS3-V1 or -V3, or PRSS3-V4 with inhibitory effects in PRSS3 knockout HCC cells. Clinically, aberrant expression of PRSS3-SVs was translated into divergent relevance in patients with HCC, in which significant epigenetic downregulation of PRSS3-V2 was seen in early HCC and was associated with favorable patient outcome.

\section{Conclusions}

Aberrant expression of divergent PRSS3-SVs disrupted by CpG methylation may integrate the effects of oncogenic PRSS3-V2 and tumor-suppressive PRSS3-V1, resulting in the molecular diversity and functional plasticity of HCC. Dysregulated expression of PRSS3-V2 by site-specific CpG methylation may have potential diagnostic value for patients with early HCC.

\section{Background}


Human primary liver cancer is one of the most lethal tumors with a dismal prognosis, featuring extensive heterogeneity and aggressiveness in the context of genetic and epigenetic aberrations [1-5]. Regardless of many approaches developed for the management of liver cancer in the past decade, its incidence and mortality rate continue to increase worldwide [1]. Liver hepatocellular carcinoma (HCC or LIHC) accounts for approximately $75-85 \%$ of all primary liver cancers. Most HCCs (>90\%) develop from chronic inflammation-induced liver cirrhosis contributed by multiple risk factors such as hepatitis viruses, alcohol consumption, and non-alcoholic fatty liver disease, which trigger the molecular complexity of intratumor heterogeneity $(\mathrm{ITH})$ increasing HCC phenotypic diversity and therapeutic resistance [1, 2].

Large-scale bioinformatics datasets generated with next-generation sequencing technologies reveal a comprehensive landscape of genomic and epigenetic heterogeneity among HCC cell lines and tissue specimens [3-7]. These studies offer invaluable insight into the molecular basis of ITH to categorize HCC into proliferative and non-proliferative subclasses in favor of integrative molecular monitoring malignant transformation and management of HCC. However, aside from most genetic alterations occurred in passenger genes that may be associated with aging and pollution, most genetic variants such as driver mutations in TP53, TERT and CTNNB1 detected in HCCs are not clinically relevant, or are not potentially targetable for the existing drugs [2]. This gives rise to a growing drive to integrate non-genetic variations into ITH, and to distinguish between functional and non-functional ITH [7, 8]. Pre-mRNA alternative splicing (AS), as a key co- and post-transcriptional process drives non-genetic phenotypic heterogeneity, disruption of which generates aberrant splice variants $(S V S)$ that contributes to ITH and functional divergence, thus functionally important to carcinogenesis and oncotherapeutics resistance [9-12].

Proteases play critical roles in multiple biological processes and are associated with a wide variety of pathological conditions, including carcinogenesis [13]. As a group of the trypsin-family serine proteases, human trypsinogen genes, protease serine 3 (PRSS3), encodes PRSS3, also called mesotrypsinogen (MTG) [14-16]. PRSS3 gives rise to four experimentally validated $S V s$, referred to as trypsinogen transcript variant 1, 2, 3, and 4 (PRSS3-V1, -V2, -V3 and -V4), encoding PRSS3 isoform 1 (also known as brain form or trypsinogen 4, TRY4) [16, 17], PRSS3-2 (isoform form C or MTG) [15, 18], PRSS3-3 (isoform form B or trypsinogen IV) [19], and PRSS3-4 (new isoform form or trypsinogen 5), respectively [20]. Other than PRSS1 and PRSS2 as the major digestive enzymes in the pancreas, PRSS3 is a minor constituent trypsin isoform but physiologically critical due to its resistance to common trypsin inhibitors [13-15]. In addition to digestive activity, PRSS3 has long been implicated in the pathogenesis of several malignancies and is therefore a promising biomarker and potential therapeutic target for cancer [21-31]. However, the functional roles associated with the expression of PRSS3 in cancer development are debatable. On one hand PRSS3 was shown to be upregulated in association with cancer metastasis, recurrence and poor prognosis [21-26, 28-31]. But on the other hand, PRSS3 was suggested as a tumor suppressor gene due to epigenetic silencing [32-36]. Although the evidence supports the dual roles of proteases in carcinogenesis depending on cellular sources and cancer microenvironment $[11-13,21-23$, $32,33,35]$, the underlying molecular basis of PRSS3 for its pro- and anti-tumorigenic roles shown in different cancer types, even reported in the same type of cancer, such as in esophageal adenocarcinoma 
$[25,34]$, lung cancer $[24,33]$ and liver cancer [28, 35], remains elusive that caused many miscellaneous aliases to PRSS3 impact its potential target-therapeutic applications $[4,12,13,22,27,35]$.

As SVs emerge as new candidates for diagnostic and prognostic biomarkers and therapeutic targets [10, 11], we systematically investigated the expression and epigenetic alteration of PRSS3-SVs functionally in relation to HCC heterogeneity. We found that differential expression of PRSS3 in HCC was attributed to aberrant expression of divergent $P R S S 3-S V S$, which was epigenetically dysregulated by site specific abnormal CpG methylation. We also found different functionality and clinical relevance of PRSS3-SVs in HCC cells and tissues. Therefore, epigenetic dysregulation of expression of PRSS3-SVs may be the molecular basis of PRSS3 to exert paradoxical effects on hepatocarcinogenesis.

\section{Materials And Methods}

\section{Data availability}

The datasets used for this study are publicly available on the websites: the Cancer Model Repository (LIMORE) (https://www.picb.ac.cn/limore/home) [6]; the Cancer Genome Atlas (TCGA, https://www.cancer.gov/) [37]; the Gene Expression Profiling Interactive Analysis (GEPIA, http://gepia.cancer-pku.cn/) [38]; the Cancer Cell Line Encyclopedia (CCLE, http://www.broadinstitute.org/ccle) [39]; the Cancer Dependency Map (DepMap, https://depmap.org/portal/, DepMap Public 20Q3)[40]; the Broad Genome Data Analysis Center (http://gdac.broadinstitute.org) [41].

\section{Cell lines}

Human HCC cell lines include well differentiated (HepG2 and Huh7) and poorly differentiated (SK-Hep-1, SMMC-7721 and LM3) cell lines and were authenticated by STR profiling. The origin and growth conditions of all cell lines used in the study were described previously [35, 42]. The cells were split to low density (30\% confluence) for overnight culture, and were then treated with $5 \mu \mathrm{M}$ of 5-aza-2'-deoxycytidine (5-aza-CR, Sigma-Aldrich, St Louis, MO, USA) for 96 hours with the medium exchanged every 24 hours.

\section{Cell line construction}

HepG2 and SK-Hep-1 cells with stably ectopic expression of PRSS3-V1, -V2, -V3 and -V4 were constructed as described [35]. CRISPR-Cas9 system was used to generate a Huh7 cell line with knockout PRSS3 $\left(P R S S 3^{K O}\right.$ ). PRSS3 ${ }^{K O}$ Huh7 cell line was then separately transfected PRSS3-V1, -V2, -V3 or -V4 construct to establish stable re-expression of $P R S S 3$ transcripts dubbed $P R S S 3^{K O+V}$ cell model. The cDNA coding human PRSS3-V1 (NM_007343.3), -V2 (NM_002771.3), -V3 (NM_001197097.2) and -V4 (NM_001197098.1) were purchased from GeneCopoeia (Rockville, MD, USA) to construct recombinant plasmid. In vitro transfection was performed using Lipofectamine 2000 (Invitrogen, Carlsbad, CA, USA) following the manufacturer's instruction. The CRISPR/ Cas9 bivector lentivirus was custom ordered from Genechem (Shanghai, China). The sgRNA was GGCACTGAGTGCCTCATCTC. 


\section{Cell viability}

HepG2, SK-Hep-1 and Huh7 cells were seeded into 96-well plates at $2 \times 10^{3}$ cells/well. Cell viability was measured everyday by using 3-(4,5-dimethylthiazol-2-yl)-2,5-diphenyltetrazolium bromide (MTT) assay Kit (KeyGEN Biotech, Jiangsu, China). The absorbance at $490 \mathrm{~nm}$ wavelength was detected using a microplate reader (Thermo Multiskan MK3, Thermo Fisher Scientific Inc., USA).

\section{Colony formation}

HCC cells were seeded in 6-well tissue culture plates (100 cells/well) in triplicate. Colonies with more than 50 cells were counted after 2 weeks. The cells were fixed with $75 \%$ ethanol for 30 minutes and stained with $0.2 \%$ crystal violet (Beyotime Ltd., Jiangsu Province, China) for 20 minutes.

\section{Transwell invasion assay}

Transwell apparatus was used with 8- $\mu$ m polyethylene terephthalate membrane filters (Corning Inc.; Corning, NY, USA). The upper chambers were seeded with $200 \mu \mathrm{l}$ of serum-free medium containing $1 \times$ $10^{4}$ of serum-starved cells. The lower chambers were filled with $500 \mu \mathrm{l}$ of $10 \%$ FBS-DMEM. After 24 hours, cells that invaded to the lower chamber were fixed and stained with $0.2 \%$ crystal violet (Beyotime) as previously described [35].

\section{RNA isolation and RT-qPCR}

Cells were harvested for RNA isolation using RNeasy Mini Kit (QIAGEN) and first strand cDNA was synthesized with the Superscript First-Strand Synthesis System (Invitrogen). RT-qPCR was performed using primers as described [35]. The relative expression level of each mRNA was normalized by $\beta$ actin using $2^{-\Delta \Delta C t}$ method

\section{Methylation-specific qPCR (MS-qPCR)}

DNA extraction, bisulfite modification and MSP-PCR were performed as described [35, 43]. Genomic DNA was extracted from tissues using the QIAamp DNA mini Kit (Qiagen) followed by quantitative analysis using NanoDrop 1000 spectrophotometer (Thermo Fisher Scientific, Inc.). Bisulfite modification of DNA was performed using Zymo DNA Methylation Kit (Zymo Research). The positive and negative template control were the Human Methylated \& Non-methylated DNA Set (Zymo Research). MSP-qPCR was performed by using methylated or unmethylated primer pairs specifically for PRSS3 [35] and $\beta$-actin [43]. The relative level of methylation and unmethylation of PRSS3 was normalized to $\beta$-actin using the 2$\Delta \Delta \mathrm{Cq}$ method.

\section{Methylated DNA immunoprecipitation-qPCR (MeDIP-qPCR)}

Genomic DNA was extracted from the HCC cells. The purified DNA is then subjected to sonicated into 200 1000bp fragments. A 10\% sonicated DNA was kept as input control. The denatured DNA fragments 
(Input fractions) were incubated with $2 \mu \mathrm{g}$ anti-5-methylcytidine $(5 \mathrm{mC})$ (Active motif, 91311) or $2 \mu \mathrm{g}$ control IgG (Sigma-Aldrich, 15006) monoclonal antibodies at $4^{\circ} \mathrm{C}$ overnight, following by precipitation using protein $A$ beads. After washing, immunoprecipitated DNA (IP fractions) and input control fraction were purified by using a QIA quick purification kit (QIAGEN, Valencia CA, USA) and analyzed by qPCR using primers as following: F: 5'- CTGTGATGGAGAGGGGGTTC -3'; R:5'- GAGTAGTGTGCGCATCGGT-3'.

\section{Statistical analysis}

The data are expressed as means \pm standard deviation (SD) of at least three independent experiments. PRSS3 transcripts expression, epigenetic alterations and associative clinicopathological correlation were analyzed by using the two-tailed Student's t-test, Wilcoxon rank sum test, one-way analysis of variance (ANOVA) with Tukey's post hoc test, Spearman rank test and Fisher's exact test, or $\chi^{2}$ or Fisher's exact tests. Cancer-related survival was analyzed using Kaplan-Meier method, and was compared using logrank tests. Statistical significance was considered when $P<0.05$. All statistical analyses were performed using SPSS version 23.0 (IBM Corp.).

\section{Results}

\section{Differentially decreased PRSS3 expression in HCC attributable to disruption of PRSS3 transcripts}

The RNAseq data from the DepMap portal demonstrated differential expression of PRSS3 in 24 human HCC cell lines (Fig. 1A, Table S1). This was further shown in $81 \mathrm{HCC}$ cell lines from the Cancer Model Repository (LIMORE) (Figure S1A). PRSS3 expression in HCC cell lines determined by RT-qPCR revealed levels of very low (PRSS3 ${ }^{L O H}$ ) to very high $\left(P R S S 3^{H i g h}\right)$ as compared to human fetal liver L02 cells (Fig. 1B). Comparative analysis using the TCGA RNAseq data from FIREHOSE Broad GDAC showed divergent features $\mathrm{s}$ of PRSS3 expression in HCC tissues compared to their matched non-tumor tissues ( $\mathrm{n}$ = 50) (Fig. 1C, Table S2), which was further evidenced from analysis of more HCC tissue specimens $($ Tumor $=371)($ Fig. 1D, Table 1). GEPIA portal combined TCGA with GTEx RNAseq datasets showed that PRSS3 expression was more varied in HCC tissues $(n=369)$ than in normal controls $(n=160)$ (Figure S1B)[37, 41], albeit with no statistical significance. PRSS3 mRNA level was lower but with an extraordinary wide range in HCC tissues as compared to normal tissues, suggesting an aberrant and differential expression of PRSS3 expression in HCC. 
Table 1

Correlation between the mRNA levels of PRSS3 transcripts with clinicopathologic characteristics in patients with HCC

\begin{tabular}{|lllll|}
\hline Characteristics & N & \multicolumn{2}{l}{ PRSS3 Expression } & P \\
\cline { 3 - 4 } & & High & Low & \\
\cline { 3 - 4 } & & N (\%) & N (\%) & \\
Total & 371 & $184(49.6)$ & $187(50.4)$ & \\
Gender & & & & \\
Male & 250 & $123(49.2)$ & $127(50.8)$ & \\
Female & 121 & $61(49.6)$ & $60(50.4)$ & 0.9137 \\
Cancer stage & & & & \\
\hline I & 171 & $82(47.9)$ & $89(52.1)$ & \\
\hline II & 86 & $43(50.0)$ & $43(50.0)$ & 0.8591 \\
\hline III & 85 & $46(54.1)$ & $39(45.9)$ & 0.4259 \\
\hline IV & 5 & $3(60.0)$ & $2(40.0)$ & 0.6736 \\
\hline Undefined & 24 & $10(41.7)$ & $14(8.3)$ & \\
\hline Tumor grade & & & & \\
\hline I & 55 & $21(38.2)$ & $34(61.8)$ & \\
\hline II & 177 & $88(49.7)$ & $89(50.3)$ & 0.1794 \\
\hline III & 122 & $67(54.9)$ & $55(45.1)$ & 0.0576 \\
\hline IV & 12 & $5(41.7)$ & $7(58.3)$ & 1 \\
\hline Undefined & 5 & $3(60.0)$ & $2(40.0)$ & \\
\hline & & & & \\
\hline
\end{tabular}

To explore the molecular basis of the divergent expression of PRSS3 in HCC, we dissected the expression of four identified PRSS3-SVs expressed in various tissues [15-20] (Fig. 2A). Analysis of the DepMap data revealed that in $24 \mathrm{HCC}$ cell lines, $P R S S 3-V 1$ and $-V 2$ were two major transcripts contributing to the expression of $P R S S 3$ because PRSS3-V3 was poorly expressed while PRSS3-V4 was absent (Fig. 2B, Table S1). RT-qPCR showed that despite almost undetectable PRSS3-V4 and very low expression of $P R S S 3-V 3$ in all cell lines, PRSS3-V1 was expressed at low levels in L02 cells, whereas PRSS3-V1 and -V2 were minimally expressed in HepG2, SK-Hep-1 and SMMC-7721 cells but highly expressed in Huh7 and LM3 cells (Fig. 2C).

Through analysis of the expression level of PRSS3-SVs in 50 paired tissue samples, we found that PRSS3V2 and also -V1 were predominantly present in both normal and tumor tissues (Fig. 2D, Table S2). 
Figure 2E showed that the expression of PRSS3-SVs was decreased in $371 \mathrm{HCC}$ tissue samples in a bipolar pattern as compared to normal liver tissues. Co-expression analysis of both HCC cell line and tissues summarized in Table 2 further showed that the highest contribution of PRSS3-SVs to PRSS3 High was co-expressed PRSS3-V2 and -V1, not by either PRSS3-V2 or -V1 in the specimens, confirming PRSS3$V 2$ was the predominant transcript in PRSS3 ${ }^{H i g h}$ in HCC. Meanwhile, $P R S S 3^{L o w}$ was also resulted from decreased expression of $P R S S 3-V 2$ and/or $-V 1$ because the minimally expressed PRSS3-V3 minimally affected the eventual expression of $P R S S 3$ despite $P R S S 3-V 3^{L O W}$ most frequently associated with $P R S S 3^{L O W}$ in HCC. These results thereby revealed disruption of $P R S S 3$ transcripts towards a bipolar expression contributing to aberrant and differential expression of PRSS3 in HCC, in which PRSS3-V2 was a dominant transcript leading to PRSS3 expression.

Table 2

Predominance and co-expression of PRSS3 transcripts in HCC cell lines and tissues

\begin{tabular}{|lllllllll|}
\hline & \multicolumn{3}{c}{ Cell lines $(\mathbf{n}=\mathbf{2 4})$} & \multicolumn{5}{c|}{ Tissue specimens $(\mathbf{n = 3 7 1})$} \\
\hline Transcript(s) & High & $\%$ & Low & $\%$ & High & $\%$ & Low & $\%$ \\
\hline PRSS3 & 12 & 100 & 12 & 100.00 & 184 & 100.00 & 187 & 100.00 \\
\hline PRSS3-V1 & 9 & 75.00 & 9 & 75.00 & 163 & 88.59 & 166 & 88.77 \\
\hline PRSS3-V2 & 10 & 83.33 & 9 & 75.00 & 173 & 94.02 & 175 & 93.58 \\
\hline PRSS3-V3 & 1 & 8.33 & 11 & 91.67 & 48 & 26.09 & 185 & 98.93 \\
\hline PRSS3-V1 + V2 & 8 & 66.67 & 6 & 50.00 & 153 & 83.15 & 156 & 83.42 \\
\hline PRSS3-V2 + V3 & 0 & 0.00 & 9 & 75.00 & 45 & 24.46 & 173 & 92.51 \\
\hline PRSS3-V1 + V3 & 1 & 8.33 & 9 & 75.00 & 46 & 25.00 & 165 & 88.24 \\
\hline PRSS3-V1 + V2 + V3 & 0 & 0.00 & 6 & 50.00 & 43 & 23.37 & 155 & 82.89 \\
\hline
\end{tabular}

Aberrant expression of divergent PRSS3 transcripts in HCC is epigenetically dysregulated by site-specific abnormal CpG methylation.

We next assessed the contribution of DNA methylation to the expression of PRSS3-SVs based on the data available from the DepMap and the FIREHOSE $[39,40]$ for three genomic regions in PRSS3. These were referred to as extended promoter region with $17 \mathrm{CpG}$ sites defined as CpG 1-17, upstream (-1000 bp upstream from the TSS of PRSS3-V1/V3 covering 2-7 of the $17 \mathrm{CpG}$ sites) and extensive fragment containing 6 CpGs (CpG A-F) (Fig. 3A, Table S4). Association analysis demonstrated an inverse association between the upstream methylation and mRNA expression of PRSS3 and its transcripts PRSS3-V1, -V2 that were distinct between hypermethylation of $P R S S 3^{L O W}\left(\mathrm{mPRSS} 3^{L O H}\right.$ ) and hypomethylation of PRSS3 ${ }^{\text {High }}$ (umPRSS3 ${ }^{\text {High }}$ ) in HCC cell lines (Fig. 3B) and tissues (Fig. 3C). 
Given that epigenetic silencing of PRSS3 has been observed in several types of human cancer [32-35], we investigated DNA methylation in the extended promoter region of PRSS3 upon the data availability (Fig. 3A). Unsupervised clustering combined with spearman correlation analysis of methylation states and expression of PRSS3 transcripts in HCC cell lines revealed that among $17 \mathrm{CpGs}$ distributing in the extended promoter region, methylation occurred at CpG site 5-17 (-89 653 bp from the TSS of PRSS3V1/V3) was reversely correlated with the mRNA expression level of PRSS3-V1, while methylation at CpG site 12-16 (522 to 564 bp to PRSS3-V1 TSS) was highly related to PRSS3-V2 expression (Fig. 3D, Figure S2, Table S5). No associative comparison was conducted on PRSS3-V3 and -V4 due to their rare expression in HCC. Moreover, despite a positive association shown in CpG site F, methylation at CpG site A-E was negatively correlated with PRSS3 expression (Fig. 3E, Figure S3). CpG site methylation at the extensive fragment of PRSS3 was decreased at site $\mathrm{A}$, increased at $\mathrm{B}, \mathrm{C}$ and $\mathrm{D}$, and then decreased at $\mathrm{E}$ and F in HCC tumors as compared to normal controls (Fig. 3F). These data suggest that an extended promoter region was important for epigenetic regulation of PRSS3 transcripts.

We then examined methylation-specific effect on PRSS3 expression using qPCR-based assays (Fig. 3A). MSP-qPCR showed hypermethylation in PRSS3 ${ }^{\text {Low }}$ cell lines (L02, HepG2, SK-Hep-1) in contrast to hypomethylation in $P R S S 3^{\text {high }}$ Huh7 cells (Fig. $3 \mathrm{G}$ ). Figure $3 \mathrm{H}$ revealed that treatment with DNA methyltransferase inhibitor 5-aza-CR caused significant upregulation of PRSS3 expression in PRSS3 ${ }^{\text {Low }}$ cell lines, but had no effect on PRSS3 ${ }^{\text {High }}$ Huh7 cells. Notably, a bipolar expression pattern was observed in $P R S S 3^{L O W}$ cell lines upon 5-aza-CR treatment showing significant upregulation of PRSS3-V1 and -V3 opposite to downregulation of $P R S S 3-V 2$, eventually integrative to the upregulation of $P R S S 3$, whereas the treatment had no effect on $P R S S 3^{\text {high }}$ Huh7 cells, actually due to integration between upregulation of PRSS3-V2 and downregulation of PRSS3-V1 or -V3. MeDIP-qPCR further showed that anti-5methylcytosine (5-mC) antibody enriched significantly less genomic DNA fragments in HepG2 cells but not in Huh7 cells upon 5-aza-CR treatment (Fig. 3I), suggesting that 5-aza-CR was effective in the expression of PRSS3 specifically by altering the DNA methylation in this target region. Although the expression of PRSS3-V3 in L02 or PRSS3-V2 in HepG2 and SK-Hep-1 cells was too low to be taken into account its decreased significance level, these results consistent with bioinformatic analysis of HCC cell lines and tissues, as well as our previous report [35], suggest that methylation occurring at this region is more critical for epigenetically controlling PRSS3 transcript activities in HCC. As shown in the summarized table (Fig. 3J), PRSS3-SVs were divergently expressed and response to 5-aza-CR treatment associated with site-specific CpG methylation that eventually determined the expression level of PRSS3 as a whole, suggesting that epigenetic dysregulation of the expression of PRSS3-SVs by site-specific CpG methylation may mediate their functional differences in HCC.

\section{PRSS3-V2 exerts oncogenic functions distinct from tumor-suppressive effects of PRSS3-V1 and -V3 in HCC cells}

The functional role of PRSS3-SVs was assessed by transfecting PRSS3-V1 to -V4 respectively into PRSS3 ${ }^{\text {Low }}$ HepG2 and SK-Hep-1 cells (defined as V1 to V4) (Fig. 4A). MTT assays showed that ectopic 
expression of $P R S S 3-V 1$ or $-V 3$ significantly inhibited HCC cell proliferation in contrast to notably enhancing effect by ectopic PRSS3-V2 expression, or in addition to unfunctional PRSS3-V4 in HCC cell proliferation as compared to the vector controls (Fig. 4B). Moreover, the results of clone formation assay showed that overexpression of PRSS3-V1 or -V3 remarkably diminished the number of colonies of HCC cells compared with the control group, but $P R S S 3-V 2$ overexpression resulted in an increased number of colonies only effectively in HepG2 cells. However, ectopic PRSS3-V4 significantly reduced clone formation in SK-Hep-1 cells but had no effect in HepG2 cells (Fig. 4C). Transwell assay further showed an inhibitory effect of PRSS3-V1 or -V3 on HCC cell migration, opposite to PRSS3-V2 that showed an enhanced effect in the cells (Fig. 4D). These results suggest a tumor-suppressive effect of PRSS3-V1/V3 versus an oncogenic effect of PRSS3-V2 in HCC cells.

To further define the phenotypic properties of PRSS3-SVs in HCC cells, we established a $P R S S 3^{K O}+V$ cell model, in which each PRSS3 transcript construct was separately transfected after endogenous PRSS3 was knocked out through CRISPR/Cas9 system (Fig. 5A). RT-qPCR showed that all the detected PRSS3 transcripts were effectively knocked out and their constructs were stably expressed in Huh7 cells, respectively designated as $P R S S 3^{K O+V 1}$ to $P R S S 3^{K O}+V 4$, or the vector control (PRSS3 ${ }^{K O+C}$ (Fig. $\left.5 \mathrm{~B}\right)$. Functional assays as shown in Fig. $5 \mathrm{C}$ to $5 \mathrm{E}$ revealed that $P R S S 3$ knockout in Huh7 cells facilitated cell proliferation, colony formation and migration, which were abolished by re-expression of PRSS3-V1 or -V3. Ectopic re-expression of $P R S S 3-V 2$ augmented the PRSS3-knockout effects on cell proliferation, colony formation, and remarkably, on migration of $P R S S 3^{K O}$ Huh7 cells. Unexpectedly, PRSS3-V4 re-expression did not affect Huh7 cell proliferation but resulted in significantly inhibition of $P R S S 3^{K O}$ Huh7 cell activity. These results demonstrate dual roles of PRSS-SVS in HCC cells and divergent disruption of PRSS3 transcripts may be integrated to establish their functional heterogeneity in HCC cells.

\section{Epigenetic alteration of PRSS3-V2 is associated with clinical relevance in patients with early HCC}

To further explore the contribution of PRSS3 transcripts to tumor heterogeneity, we used TCGA dataset to analyze their clinical relevance. We found that the expression of PRSS3 and PRSS3-V2 was similarly downregulated but with a gradually increased tendency in HCC tumors compared with control tissues, following the progression of tumors stages (Fig. 6A) and pathological grades (Fig. 6B), in which PRSS3$V 2^{\text {Low }}$ was significantly detected in tumors of early HCC patients in contrast to PRSS3-V2 ${ }^{\text {High }}$ in advanced tumors. Kaplan-Meier (K-M) analysis revealed that PRSS3-V2 ${ }^{L O W}$ was a favorable factor for overall survival of HCC patients based on cancer stages (Fig. 6C) and grades (Fig. 6D), in which PRSS3-V2 ${ }^{\text {Low }}$ patient groups with low-grade tumors showed significantly favorable outcome $(P=0.011)$. Moreover, divergent disruption of $\mathrm{CpG}$ site methylation (A to $\mathrm{F}$ ) was shown throughout clinical progression of tumors but occurred more frequently and significantly in tumors of HCC patients with early-stage (Fig. 6E) and lower-grade tumors (Fig. 6F). In such tumors alteration in $\mathrm{CpG}$ methylation at site $\mathrm{D}$ was most reversely correlated with the expression of PRSS3 and PRSS-V2. Since the region located at site D was shown as an important regulatory region specifically for epigenetic regulation of $P R S S 3$ transcripts (Fig. 3), the data suggest that site-specific epigenetic alteration of PRSS3-V2 in HCC tissues was distinct 
between mPRSS3-V2 $2^{L O W}$ in early HCC and umPRSS3 $3^{H i g h}$ in advanced HCC patients, in which early HCC patients with $P R S S 3-V 2^{L O W}$ tumors had better outcomes.

\section{Discussion}

Paradoxical effects of many genes have been observed during tumorigenesis $[13,44,45]$. In this study we explored the dysregulation of splicing variants expression functionally contributing to HCC heterogeneity. Protease PRSS3 is the first to link the enzyme to prostate cancer leading to the development of a compound to stop PRSS3 from promoting metastasis $[13,46]$. Since the high similarity in both sequences and structures to different trypsinogen isoenzymes made it difficult to delineate their functionally associated transcripts distributed in different tissues [13, 14], the protumor [21-31] or antitumor properties of PRSS3 [32-36] were deciphered relying on the cellular source and cancer microenvironment $[13,14,35]$. In this study, we found differentially expressed PRSS3 in HCC due to CpG methylation-mediated epigenetic dysregulation of its splice variants. Different PRSS3-SVs expressed in HCC showed a dual role in hepatocarcinogenesis that may increase phenotypic diversity. Our study uncovered an epigenetic-mediated PRSS3 transcript variance contributing to non-genetic phenotypic diversity of HCC [44]. To our best knowledge, this is the first study of functional dissection of the expression of PRSS3-SVs in cancer and thus has important implications in HCC patient-tailored management.

PRSS3 is known as a digestive protease with restricted expression in pancreas. However, the preferential expression of PRSS3-SVs differs in human tissues suggests a tissue-selective expression manner. For instance, $P R S S 3-V 2$ was exclusively expressed in human pancreatic tissue and fluid encoding MTG [16, 47]. Canonical PRSS3-V1 was originally identified in human brain $[17,47]$. PRSS3-V3 shares a same TSS with PRSS3-V1 but has a different in-frame exon with deduced a 261-amino acid sequence (formerly named isoform B) [19]. PRSS3-V4 was cloned from keratinocytesand shown in participating keratinocyte terminal differentiation [20]. Our study showed the differential expression of PRSS3 as a DEG in HCC across a large expression range that could be used to phenotypically distinguish between $P R S S 3^{L O W}$ and $P R S S 3^{H i g h} \mathrm{HCC}$ cells and tissues. Accordingly, we found divergent expression of PRSS3-SVs towards bipolarity following clinical progress from downregulation in early HCC to upregulation in advanced cancer, unveiling the molecular basis of PRSS3 in tissue-selective expression of its splice transcripts in HCC. Despite the infrequent or minimal expression of PRSS3-V3 and unexpressed PRSS3-V4, the divergent expression changes of $P R S S 3-V 2$ and/or - V1 were major contributors to the transcript heterogeneity of PRSS3 in HCC. Notably, the expression of PRSS3-SVs was dynamically altered following clinical progress from downregulation in early HCC to upregulation in advanced cancer. PRSS3 transcript heterogeneity was further evidenced by its divergent responses to 5-aza-CR treatment of HCC cells, distinguishing between upregulation of PRSS3-V1 or -V3 but downregulation of PRSS3-V2 in PRSS3Low HCC and downregulation of PRSS3-V1 but upregulation of PRSS3-V2 in PRSS3 ${ }^{H i g h}$ HCC. The divergent expression of PRSS3 transcripts and their responding to 5-aza-CR prompted our consideration of the effects of non-genetic heterogeneity on chemotherapy-response, because this well-known anticancer drug 
has broad clinical applications and the mis-splicing regulation as non-genetic mechanisms is frequently linked to therapy escape [48-50]. For precise evaluation of the clinical effectiveness and drug resistance by using a DEG, its functional splice variants, rather than its overall expression, need to be taken into account. Nevertheless, it was clear that differentially expressed PRSS3 decreased as a whole was mainly attributable to its aberrant transcript variance expressed in HCC.

PRSS3 translocates from chromosome 7q34, the locus of PRSS1 and PRSS2, to chromosome 9p11.2, a region frequently containing alterations $[13,51]$. However, frequent genetic variations occurred in $P R S S 3$ have not yet demonstrated disease-associated PRSS3 variants

(https://www.nextprot.org/entry/NX_P35030/medical). Alternative splicing forms dynamic interactome offering precise therapeutic approaches to correcting cancer-specific defects caused by mis-splicing regulation, in which epigenetics plays an essential role $[9,11,12,49,52-54]$. Our previously study showed epigenetic silencing of PRSS3 in HCC [35], we reasoned epigenetic regulation of PRSS3-SVS contributing to non-genetic heterogeneity in HCC. The different TSSs and start codes in PRSS3 suggest that $P R S S 3$, like the majority of protein-coding genes, tends to be regulated by multiple or alternative promoters, the usage of which provides a pre-transcriptional control of gene activity to express its different isoforms in a tissue-specific manner $[4,6,11,25]$. Here, we found an extended promoter region covering the upstream and the intragenic region of $P R S S 3-V 1 / V 3$ and $-V 4$, providing a site-specific way to regulate the expression of $P R S S 3-S V$. Both HCC cells and tissues were phenotypically classified as $\mathrm{mPRSS3}{ }^{L O W}$ and $\mathrm{umPRSS3} 3^{\text {High }}$ based on CpG methylation in association with expression of PRSS3 transcripts. Compared to the consistent upstream hypermethylation, site-specific CpG methylation in the intragenic region was found more associated with the expression of $P R S S 3-V 1$ and $V 2$, suggesting that this extended promoter region played a central role in regulation of both PRSS3-V1 and V2. Given that epigenetic promoter alterations can change chromatin accessibility of transcription regulatory elements binding to transcription factors $[9,12,44,52,55-57]$, the upstream hypermethylation of $P R S S 3$ may impact tissue-specific cis-regulatory modules that may alter transcription activity of PRSS3-SVs in HCC. Dynamic disruption of different $\mathrm{CpG}$ site methylation within the extended promoter region may affect certain transcriptional regulators or splicing factors occupancy, resulting in an alternation in exon skipping to control the expression of PRSS3-V1 or -V3. Meanwhile, site-specific epigenetic control of $P R S S 3-V 2$ suggests that the extended promoter may be a distal regulatory region in regulation of $P R S S 3-$ $V 2$ through a very different epigenetic pathway [58]. Consistent with this, epigenetic silencing of PRSS3 was found in several cancer types and our previous study showed an intragenic DNA methylation within the extended promoter region contributing to PRSS3 downregulation in HCC [35]. This study was the first to dissect epigenetic heterogeneity in regulation of PRSS3-SVs that may provide important implications for understanding epigenetic contributions to the genomic occupancy of transcription factors during transcription, in which many events may appear to be co-spliced with distant events[53, 55-57] .

Many transcript isoforms can exist per gene [9-11], most of which are thought not to be functionally relevant [59]. Recently, comprehensive gain- and loss-of function works had shown the functional importance of SVs in tumor heterogeneity by linking genetic variants to individual's phenotypes [52-54, 
$60,61]$. PRSS3 appears to be transcribed differentially to display heterogenous functions in cancer, in which a dual role or contradictory effects reported might be due to MTG (PRSS3-V2) to be functionally regarded as PRSS3 $[13,14,21,22,24]$. We hereby deciphered a functional difference among the PRSS3 isoforms by using constructed Huh7 cell model. Despite PRSS3-V2 /MTG-mediated an oncogenic effect in HCC in line with the pro-malignancy activities of MTG shown in other cancer types[13, 14, 21, 22, 24], PRSS3-V1 or -V3 were found as tumor-suppressors in HCC cells, while ectopic PRSS3-V4 showed an inhibitory effect on the PRSS3 ${ }^{k o}$ Huh7 cells. $P R S S 3^{k o}$ resulting in pro-tumor effects in Huh7 cells suggests a tumor-suppressive role of PRSS3 played in HCC that was attributed to the co-expressed PRSS3-V1 and $V 2$, the two isoforms with opposite functionality. This is in line with our previous observations [35] and may explain some but not all cases of a similar phenotype with well-differentiated and/or low metastatic potential appearing in either PRSS3 ${ }^{\text {Low }}$ (e.g. HepG2, SK-Hep1 cells) or PRSS3 ${ }^{\text {High }}$ (Huh7 cells) live cancer cell lines, or a dual role of $P R S S 3$ contradictorily shown in carcinogenesis. To support this, corresponding clinicopathological analysis of HCC specimens compared to the normal tissue controls uncovered that $P R S S 3-V 1$ and - V2 were main functional components of clinical relevance since PRSS3-V1 and - V2 were bipolarly presented in both PRSS3 ${ }^{L O W}$ or $P R S S 3^{H i g h}$ tissues, thereby their abnormal co-expression could bring out functional heterogeneity including insignificant or paradoxical clinical association. However, a signature pattern of epigenetic regulation of PRSS3 expression by site-specific CpG methylation showed dynamically from $\mathrm{mPRSS} 3^{L O W}$ to umPRSS3 ${ }^{\text {High }}$ through clinical progression, better matched to PRSS3-V2, suggesting PRSS3-V2 to be a more prevalent isoform functionally through clinical progression of HCC. Accordingly, significant epigenetic downregulation of $P R S S 3-V 2$ was seen in early HCC with favorable patient outcome. This supports an oncogenic role of PRSS3-V2/MTG dominantly in HCC thus providing early diagnostic and prognostic value for $\operatorname{HCC}[14,21,22,24]$. Thus, our study provides additional evidence for supporting the hypothesis of functional hepato-heterogeneity attributed to genetic and epigenetic factors [3-6].

Aberrant expression of SVs in cancer generates tumor functional heterogeneity conducting eventual cellular phenotype(s) or influence cell fate determination $[4,5,7,8]$. In this regard, delineation of the heterogeneity of $P R S S 3$ expression and epigenetic regulation is critical for clarifying the molecular basis of PRSS3 transcripts thus facilitating functional interpretation of the paradoxical effects PRSS3 exerting in cancer development. Functional classification and experimental dissection of PRSS3-SVs and their response to 5-aza-CR treatment distinct between $P R S S 3^{L O W}$ and $P R S S 3^{H i g h} \mathrm{HCC}$ cells (such as Huh7 versus HepG2 cells) may be used as an experimental model for studying PRSS3 splicing-mediated functional heterogeneity during hepatocarcinogenesis. In contrast to permanent genetic mutations, epigenetic disruptions frequently occurred in early clinical stages and plays an important role in modulating cell malignancy in a progressive and reversible manner. Therefore, delineation of the precise molecular mechanisms underlying epigenetic regulation of PRSS3-SVs could contribute to molecular phenotypes of HCC.

This study on bioinformatic analysis of RNA sequencing data of PRSS3-SVs and their clinical relevance gave lots of insignificantly divergent results. For instance, $P R S S 3^{L O W}$ was shown in 50 paired HCC tissues, 
consistent with our previous observation [35] and the analyses showing in the TCGA and the UALCAN portal [37]. But its decrease was no longer statistically significant in more HCC tissue specimens, due to different statistical methods, or integration of the RNAseq data with different median cutoff values for extensively divergent expression of PRSS3-SVS in HCC specimens. Therefore, the conventional parameter such as the median cutoff values may need to be reevaluated for grouping a DEG with divergent expression levels. Moreover, the functional heterogeneity could be caused by microenvironment enhanced co-expression diversity of PRSS3-SVs. As a result, further studies with larger sample size of paired HCC specimens are warranted to validate our observations.

\section{Conclusions}

In summary, PRSS3 was aberrantly expressed in HCC due to epigenetic dysregulation that was integrated with divergent expression of PRSS3-SVs by site-specific CpG methylation. The effects of oncogenic $P R S S 3-V 2$ and tumor-suppressive PRSS3-V1 in HCC cells may increase the molecular diversity and functional plasticity of hepatocarcinogenesis. Epigenetic dysregulation of PRSS3-V2 distinct between $m P R S S 3-V 2^{L O W}$ in early clinical stages and umPRSS3 ${ }^{H i g h}$ in advanced tumors has potential diagnostic value for patients with early HCC.

\section{Abbreviations}

5-Aza-CR 5- aza-2'-deoxycytidine

CCLE the Cancer Cell Line Encyclopedia

DepMap the Cancer Dependency Map

FPKM Fragments Per Kilobase Million

GTEx the Genotype-Tissue Expression

GEPIA the Gene Expression Profiling Interactive Analysis

HCC Hepatocellular carcinoma

LIHC Liver hepatocellular carcinoma

mPRSS3Low lowly-expressed PRSS3-SVs with hypermethylation

MTG Trypsin-3 isoform 2/mesotrypsinogen;

PRSS3 Serine protease 3 gene

PRSS3 Serine protease 3 
PRSS3High or PRSS3Low highly- or lowly- expressed PRSS3

PRSS3-V1 -V4 PRSS3 transcript variant $1,-2,-3$ and -4

PRSS3-SVs splice transcripts of PRSS3

TCGA The Cancer Genome Atlas

TPM Transcripts Per Million

TRY4 trypsin-3 isoform 1/trypsinogen 4

umPRSS3High highly-expressed PRSS3-SVs with hypomethylation

\section{Declarations}

Ethics approval and consent to participate: Not applicable.

Consent for publication: All authors read and confirmed that this work can be published.

Availability of data and materials: All data are publicly released from TCGA, GTEX, GDAC, GEPIA, FIREHOSE and CCLE databases and hyper-links including citations have been included in the "Materials and Methods" and "Result" section.

Competing interests: The authors declare that they have no competing interests.

Funding: This study was funded by the Scientific Research Project of Beijing Educational Committee (Grant No. KM202110025004), the Intramural Research Funding Program from Beijing Tuberculosis and Thoracic Tumor Research Institute/Beijing Chest Hospital, National Key Research and Development Program of China (2018YFA0208902, 2020YFC2002705); Beijing Natural Science Foundation of China (BJSFC No. 7171008,7214242); National Science Foundation of China (NSFC Grant No. 81872021, U1604281, 81672138); National Key Scientific Instrument Special Program of China (Grant No. 2011YQ03013405). KC, JH and JMW were also funded in part by Federal funds from the National Cancer Institute, National Institutes of Health, under Contract No. HHSN261200800001E and were supported in part by the Intramural Research Program of the NCl, CCR, LCIM, NIH.

Authors' contributions: Conceptualization, SL, HX and JH; Formal analysis, SL, HX, MP, XG, XCZ, LZ, FJ, $\mathrm{YH}, \mathrm{WW}, \mathrm{JR}$ and $\mathrm{JH}$; Funding acquisition, JMW, MG and JH; Investigation, SL, HX, XZ, MP, LZ, XG, YP and ZY; Methodology, SL, XZ, HX, MP, XW, BL, RT, XCZ, ZY and JH; Resources, KC and WG; Supervision, MG. and $\mathrm{JH}$; Writing - original draft, $\mathrm{SL}$ and $\mathrm{JH}$; Writing-review \& editing, JMW and $\mathrm{JH}$. All au-thors have reviewed and agreed to the final version of the manuscript.

Acknowledgements: We thank all of our laboratory members for technical help and helpful discussions. 


\section{References}

1. Sung H, Ferlay J, Siegel RL, Laversanne M, Soerjomataram I, Jemal A, Bray F: Global Cancer Statistics 2020: GLOBOCAN Estimates of Incidence and Mortality Worldwide for 36 Cancers in 185 Countries. CA Cancer J Clin2021, 71(3):209-249.

2. Llovet JM, Montal R, Sia D, Finn RS: Molecular therapies and precision medicine for hepatocellular carcinoma. Nature reviews Clinical oncology2018, 15(10):599-616.

3. Lin DC, Mayakonda A, Dinh HQ, Huang P, Lin L, Liu X, Ding LW, Wang J, Berman BP, Song EWet al: Genomic and Epigenomic Heterogeneity of Hepatocellular Carcinoma. Cancer Res2017, 77(9):2255-2265.

4. Comprehensive and Integrative Genomic Characterization of Hepatocellular Carcinoma. Cel/2017, 169(7):1327-1341.e1323.

5. Dhanasekaran R: Deciphering Tumor Heterogeneity in Hepatocellular Carcinoma (HCC)-Multi-Omic and Singulomic Approaches. Seminars in liver disease2021, 41(1):9-18.

6. Qiu Z, Li H, Zhang Z, Zhu Z, He S, Wang X, Wang P, Qin J, Zhuang L, Wang Wet al: A Pharmacogenomic Landscape in Human Liver Cancers. Cancer cel/2019, 36(2):179-193.e111.

7. Nam AS, Chaligne R, Landau DA: Integrating genetic and non-genetic determinants of cancer evolution by single-cell multi-omics. Nature reviews Genetics2021, 22(1):3-18.

8. Chan LK, Tsui YM, Ho DW, Ng IO: Cellular heterogeneity and plasticity in liver cancer. Seminars in cancer biology 2021.

9. Sonneveld S, Verhagen BMP, Tanenbaum ME: Heterogeneity in mRNA Translation. Trends in cell biology2020, 30(8):606-618.

10. Bonnal SC, López-Oreja I, Valcárcel J: Roles and mechanisms of alternative splicing in cancer implications for care. Nature reviews Clinical oncology2020, 17(8):457-474.

11. Dvinge $H$, Guenthoer J, Porter PL, Bradley RK: RNA components of the spliceosome regulate tissueand cancer-specific alternative splicing. Genome research2019, 29(10):1591-1604.

12. Zhang J, Zhang YZ, Jiang J, Duan CG: The Crosstalk Between Epigenetic Mechanisms and Alternative RNA Processing Regulation. Frontiers in genetics2020, 11:998.

13. Lópezotín C, Matrisian LM: Emerging roles of proteases in tumour suppression. 2007, 7(10):800-808.

14. Salameh MA, Radisky ES: Biochemical and structural insights into mesotrypsin: an unusual human trypsin. Int J Biochem Mol Biol2013, 4(3):129-139. 
15. Rinderknecht H, Renner IG, Abramson SB, Carmack C: Mesotrypsin: a new inhibitor-resistant protease from a zymogen in human pancreatic tissue and fluid. Gastroenterology1984, 86(4):681-692.

16. Nyaruhucha $\mathrm{CN}$, Kito M, Fukuoka SI: Identification and expression of the CDNA-encoding human mesotrypsin(ogen), an isoform of trypsin with inhibitor resistance. The Journal of biological chemistry1997, 272(16):10573-10578.

17. Wiegand U, Corbach S, Minn A, Kang J, Müller-Hill B: Cloning of the cDNA encoding human brain trypsinogen and characterization of its product. Gene1993, 136(1-2):167-175.

18. Tóth J, Siklódi E, Medveczky P, Gallatz K, Németh P, Szilágyi L, Gráf L, Palkovits M: Regional distribution of human trypsinogen $\mathbf{4}$ in human brain at mRNA and protein level. Neurochemical research2007, 32(9):1423-1433.

19. Cottrell GS, Amadesi S, Grady EF, Bunnett NW: Trypsin IV, a novel agonist of protease-activated receptors 2 and 4. The Journal of biological chemistry2004, 279(14):13532-13539.

20. Nakanishi J, Yamamoto M, Koyama J, Sato J, Hibino T: Keratinocytes Synthesize Enteropeptidase and Multiple Forms of Trypsinogen during Terminal Differentiation. Journal of Investigative Dermatology2010, 130(4):944.

21. Jiang G, Cao F, Ren G, Gao D, Bhakta V, Zhang Y, Cao H, Dong Z, Zang W, Zhang Set al: PRSS3 promotes tumour growth and metastasis of human pancreatic cancer. Gut2010, 59(11):1535-1544.

22. Hockla A, Miller E, Salameh MA, Copland JA, Radisky DC, Radisky ES: PRSS3/mesotrypsin is a therapeutic target for metastatic prostate cancer. Molecular cancer research : MCR2012, 10(12):15551566.

23. Ghilardi C, Silini A, Figini S, Anastasia A, Lupi M, Fruscio R, Giavazzi R, Bani MR: Trypsinogen 4 boosts tumor endothelial cells migration through proteolysis of tissue factor pathway inhibitor-2. Oncotarget2015, 6(29):28389-28400.

24. Ma H, Hockla A, Mehner C, Coban M, Papo N, Radisky DC, Radisky ES: PRSS3/Mesotrypsin and kallikrein-related peptidase 5 are associated with poor prognosis and contribute to tumor cell invasion and growth in lung adenocarcinoma. Sci Rep2019, 9(1):1844.

25. Han S, Lee CW, Trevino JG, Hughes SJ, Sarosi GA, Jr.: Autocrine extra-pancreatic trypsin 3 secretion promotes cell proliferation and survival in esophageal adenocarcinoma. PloS one2013, 8(10):e76667.

26. Ma R, Ye X, Cheng H, Ma Y, Cui H, Chang X: PRSS3 expression is associated with tumor progression and poor prognosis in epithelial ovarian cancer. Gynecologic oncology2015, 137(3):546-552.

27. Radisky ES: PRSS3/mesotrypsin in prostate cancer progression: implications for translational medicine. Asian journal of andrology2013, 15(4):439-440. 
28. Terada T, Ohta T, Minato H, Nakanuma Y: Expression of pancreatic trypsinogen/trypsin and cathepsin B in human cholangiocarcinomas and hepatocellular carcinomas. Human pathology1995, 26(7):746-752.

29. Qian L, Gao X, Huang H, Lu S, Cai Y, Hua Y, Liu Y, Zhang J: PRSS3 is a prognostic marker in invasive ductal carcinoma of the breast. Oncotarget2017, 8(13):21444-21453.

30. Schaid DJ, McDonnell SK, FitzGerald LM, DeRycke L, Fogarty Z, Giles GG, MacInnis RJ, Southey MC, Nguyen-Dumont T, Cancel-Tassin Get al: Two-stage Study of Familial Prostate Cancer by Whole-exome Sequencing and Custom Capture Identifies 10 Novel Genes Associated with the Risk of Prostate Cancer. European urology2021, 79(3):353-361.

31. Aboulouard S, Wisztorski M, Duhamel M, Saudemont P, Cardon T, Narducci F, Lemaire AS, Kobeissy F, Leblanc E, Fournier let al: In-depth proteomics analysis of sentinel lymph nodes from individuals with endometrial cancer. Cell reports Medicine2021, 2(6):100318.

32. Marsit CJ, Houseman EA, Schned AR, Karagas MR, Kelsey KT: Promoter hypermethylation is associated with current smoking, age, gender and survival in bladder cancer. Carcinogenesis2007, 28(8):1745-1751.

33. Marsit CJ, Chinedu O, Hadi D, Kelsey KT: Epigenetic silencing of the PRSS3 putative tumor suppressor gene in non-small cell lung cancer. Molecular Carcinogenesis2010, 44(2):146-150.

34. Keishi Y, Koshi M, Hiroshi I, Masaki M, David S: A tumor-suppressive role for trypsin in human cancer progression. Cancer Research2003, 63(20):6575-6578.

35. Lin B, Zhou X, Lin S, Wang X, Zhang M, Cao B, Dong Y, Yang S, Wang JM, Guo Met al: Epigenetic silencing of PRSS3 provides growth and metastasis advantage for human hepatocellular carcinoma. Journal of molecular medicine (Berlin, Germany)2017, 95(11):1237-1249.

36. Marsit CJ, Karagas MR, Schned A, Kelsey KT: Carcinogen exposure and epigenetic silencing in bladder cancer. Annals of the New York Academy of Sciences2006, 1076:810-821.

37. Uhlen M, Zhang C, Lee S, Sjöstedt E, Fagerberg L, Bidkhori G, Benfeitas R, Arif M, Liu Z, Edfors Fet al: A pathology atlas of the human cancer transcriptome. Science (New York, NY)2017, 357(6352).

38. Tang Z, Li C, Kang B, Gao G, Li C, Zhang Z: GEPIA: a web server for cancer and normal gene expression profiling and interactive analyses. Nucleic acids research2017, 45(W1):W98-w102.

39. Ghandi M, Huang FW, Jané-Valbuena J, Kryukov GV, Lo CC, McDonald ER, 3rd, Barretina J, Gelfand ET, Bielski CM, Li Het al: Next-generation characterization of the Cancer Cell Line Encyclopedia. Nature2019, 569(7757):503-508.

40. Ben-David U, Siranosian B, Ha G, Tang H, Oren Y, Hinohara K, Strathdee CA, Dempster J, Lyons NJ, Burns Ret al: Genetic and transcriptional evolution alters cancer cell line drug response. Nature2018, 
560(7718):325-330.

41. Deng M, Brägelmann J, Kryukov I, Saraiva-Agostinho N, Perner S: FirebrowseR: an R client to the Broad Institute's Firehose Pipeline. Database : the journal of biological databases and curation2017, 2017.

42. Guo Y, Peng Y, Gao D, Zhang M, Yang W, Linghu E, Herman JG, Fuks F, Dong G, Guo M: Silencing HOXD10 by promoter region hypermethylation activates ERK signaling in hepatocellular carcinoma. Clin Epigenetics2017, 9:116.

43. Lin S, Wang X, Pan Y, Tian R, Lin B, Jiang G, Chen K, He Y, Zhang L, Zhai Wet al: Transcription Factor Myeloid Zinc-Finger 1 Suppresses Human Gastric Carcinogenesis by Interacting with Metallothionein 2A. Clinical cancer research : an official journal of the American Association for Cancer Research2019, 25(3):1050-1062.

44. Li Y, McGrail DJ, Xu J, Mills GB, Sahni N, Yi S: Gene Regulatory Network Perturbation by Genetic and Epigenetic Variation. Trends in biochemical sciences2018, 43(8):576-592.

45. Belluti S, Rigillo G, Imbriano C: Transcription Factors in Cancer: When Alternative Splicing Determines Opposite Cell Fates. Cells2020, 9(3).

46. Cohen I, Coban M, Shahar A, Sankaran B, Hockla A, Lacham S, Caulfield TR, Radisky ES, Papo N: Disulfide engineering of human Kunitz-type serine protease inhibitors enhances proteolytic stability and target affinity toward mesotrypsin. The Journal of biological chemistry2019, 294(13):5105-5120.

47. Tani T, Kawashima I, Mita K, Takiguchi Y: Nucleotide sequence of the human pancreatic trypsinogen III cDNA. Nucleic acids research1990, 18(6):1631.

48. Ramesh V, Ganesan K: Integrative functional genomic analysis unveils the differing dysregulated metabolic processes across hepatocellular carcinoma stages. Gene2016, 588(1):19-29.

49. Urbanski LM, Leclair N, Anczuków O: Alternative-splicing defects in cancer: Splicing regulators and their downstream targets, guiding the way to novel cancer therapeutics. Wiley interdisciplinary reviews RNA2018, 9(4):e1476.

50. Duchmann M, Itzykson R: Clinical update on hypomethylating agents. International journal of hematology2019, 110(2):161-169.

51. Rowen L, Williams E, Glusman G, Linardopoulou E, Friedman C, Ahearn ME, Seto J, Boysen C, Qin S, Wang Ket al: Interchromosomal segmental duplications explain the unusual structure of PRSS3, the gene for an inhibitor-resistant trypsinogen. Molecular biology and evolution2005, 22(8):1712-1720.

52. Gopi LK, Kidder BL: Integrative pan cancer analysis reveals epigenomic variation in cancer type and cell specific chromatin domains. Nature communications2021, 12(1):1419. 
53. Sharma A, Merritt E, Hu X, Cruz A, Jiang C, Sarkodie H, Zhou Z, Malhotra J, Riedlinger GM, De S: NonGenetic Intra-Tumor Heterogeneity Is a Major Predictor of Phenotypic Heterogeneity and Ongoing Evolutionary Dynamics in Lung Tumors. Cell reports2019, 29(8):2164-2174.e2165.

54. Calviello L, Hirsekorn A, Ohler U: Quantification of translation uncovers the functions of the alternative transcriptome. Nature structural \& molecular biology2020, 27(8):717-725.

55. Yang X, Han H, De Carvalho DD, Lay FD, Jones PA, Liang G: Gene body methylation can alter gene expression and is a therapeutic target in cancer. Cancer cel/2014, 26(4):577-590.

56. Demircioğlu D, Cukuroglu E, Kindermans M, Nandi T, Calabrese C, Fonseca NA, Kahles A, Lehmann KV, Stegle O, Brazma Aet al: A Pan-cancer Transcriptome Analysis Reveals Pervasive Regulation through Alternative Promoters. Cel/2019, 178(6):1465-1477.e1417.

57. Gomez L, Odom GJ, Young JI, Martin ER, Liu L, Chen X, Griswold AJ, Gao Z, Zhang L, Wang L: coMethDMR: accurate identification of co-methylated and differentially methylated regions in epigenomewide association studies with continuous phenotypes. Nucleic acids research2019, 47(17):e98.

58. Schoenfelder S, Fraser P: Long-range enhancer-promoter contacts in gene expression control. Nature reviews Genetics2019, 20(8):437-455.

59. Gallego-Paez LM, Bordone MC, Leote AC, Saraiva-Agostinho N, Ascensão-Ferreira M, Barbosa-Morais NL: Alternative splicing: the pledge, the turn, and the prestige : The key role of alternative splicing in human biological systems. Human genetics2017, 136(9):1015-1042.

60. Louadi Z, Yuan K, Gress A, Tsoy O, Kalinina OV, Baumbach J, Kacprowski T, List M: DIGGER: exploring the functional role of alternative splicing in protein interactions. Nucleic acids research2021, 49(D1):D309-d318.

61. Enhancing GTEx by bridging the gaps between genotype, gene expression, and disease. Nature genetics2017, 49(12):1664-1670.

\section{Figures}


a

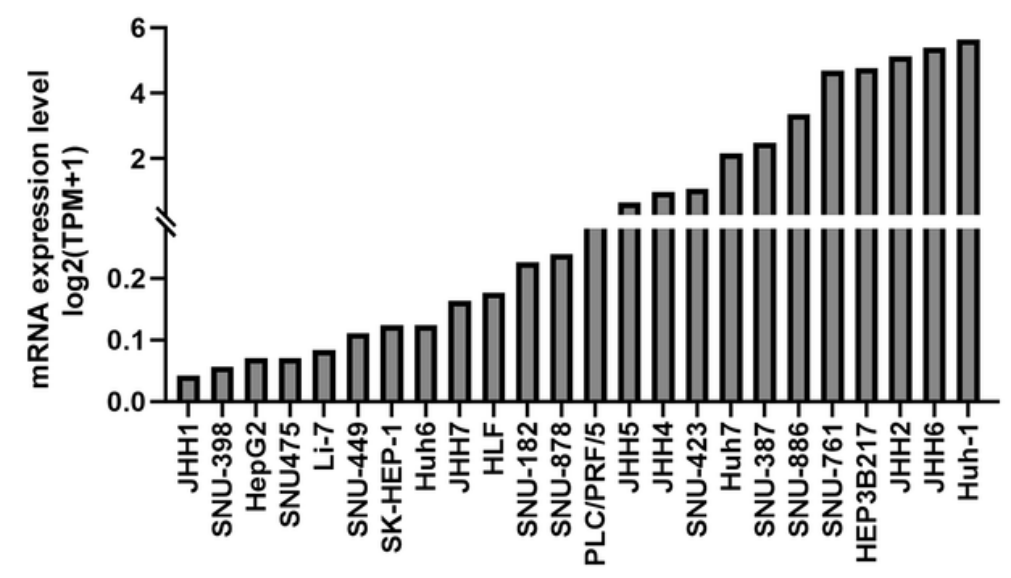

C

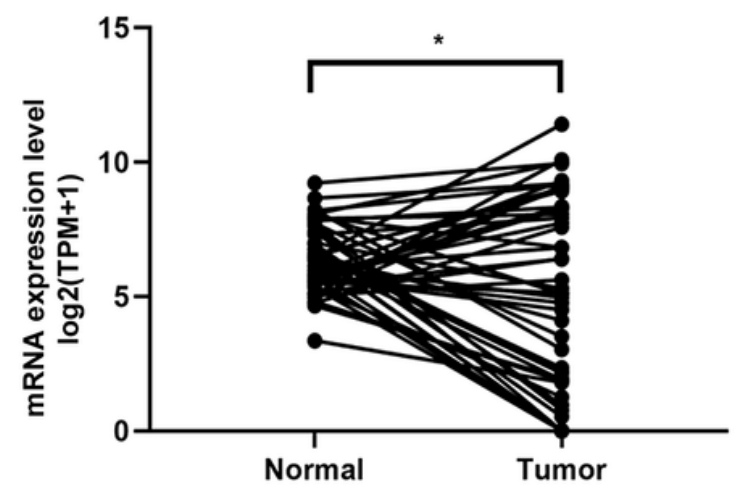

b

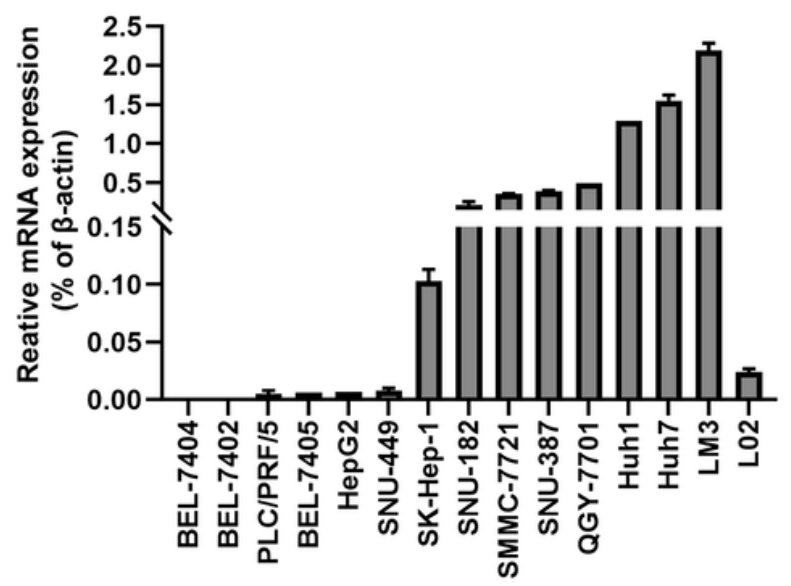

d

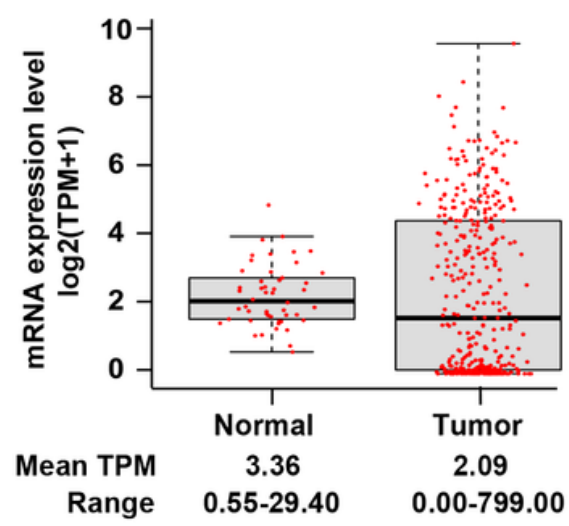

Figure 1

PRSS3 expression in human HCC cell lines and tissues. (A) The mRNA level of PRSS3 expression in 24 HCC cell lines using RNAseq data extracted from the DepMap website. The expression bar chart is sorted by PRSS3 mRNA expression levels processed as log2 (TPM+1) scale. TPM: Transcripts Per Million. (B) RT-qRCR analysis of PRSS3 expression in $14 \mathrm{HCC}$ cell lines and human fetal liver cell line L02. The relative expression of PRSS3 mRNA was normalized with $\beta$-actin presented as the mean \pm SD from three independent experiments done in triplicate. (C-D) The RNAseq data was sourced from the FIREHOSE (Table 1). PRSS3 expression in 50 pairs of tumors and matched solid normal tissues from patients with HCC (C), or extensively in 371 tumor samples versus 50 normal tissues from HCC patients (D). ${ }^{*}<0.05$, by Student $t$ test. 
a

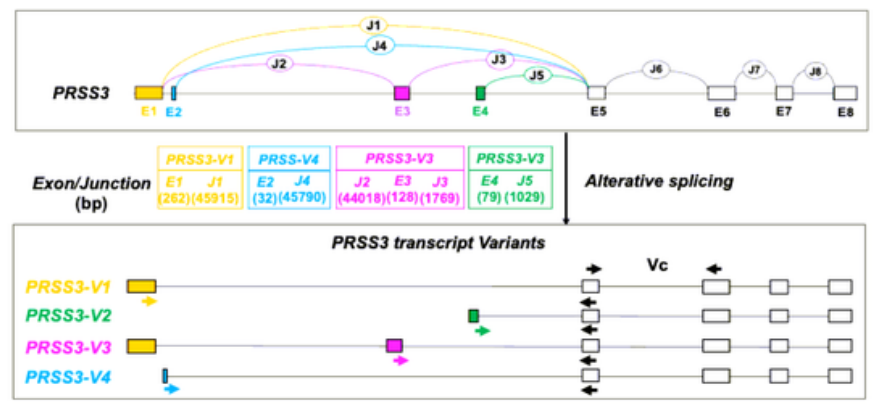

b

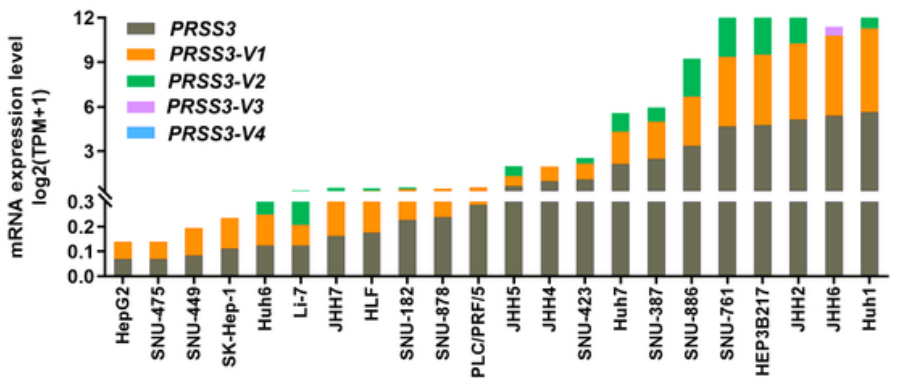

C
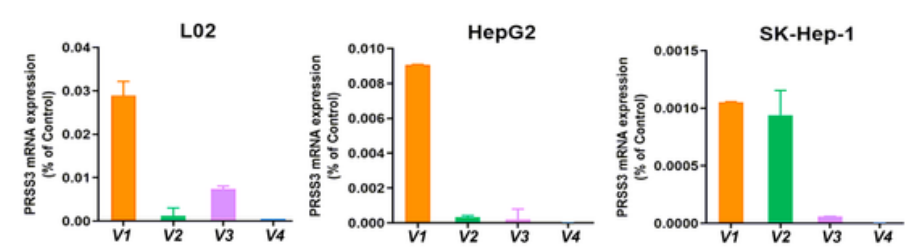

d

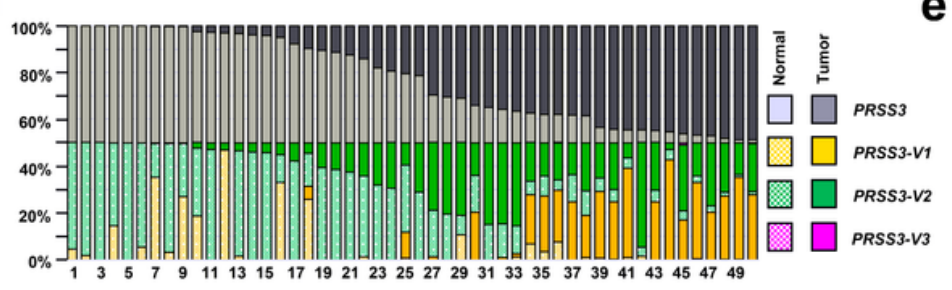

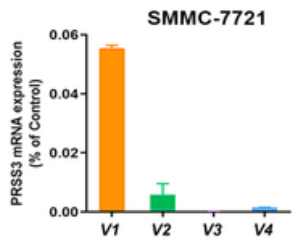
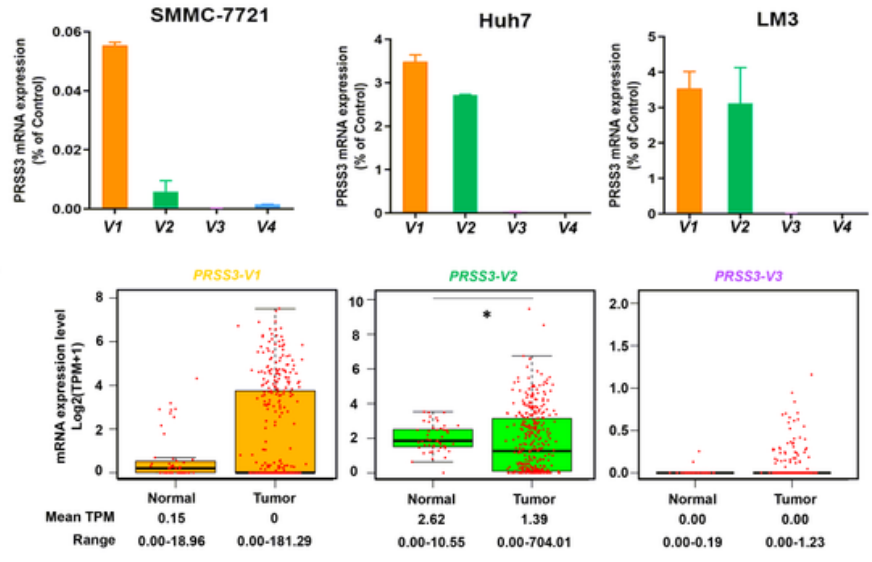

\section{Figure 2}

The expression of PRSS3 splice variants in human HCC cells and tissues. (A) A schematic overview of the human PRSS3 gene structures and its splicing transcript variants, and the RT-qPCR primers designed. The top diagram represents the genomic organization of the PRSS3 gene. Alternative splicing within the 5 region of PRSS3 gene leads to the creation of PRSS3-V1 $\sim$-V4. The exons and introns are represented as boxes and lines (not drawn to scale). E1-E8: Exons; J1-J8: Junctions. E5-8: gray boxes common to all four variants. E1 to 4: sequence-specific for PRSS3-V1 (brown), -V4 (blue), -V3 (purple), and -V2 (green), respectively. Arrowheads indicate primer sets locations used for amplification of PRSS3-SVs. Forward primers were designed specifically for PRSS3-SVs. Reverse primer was common to all. Vc: RT-qPCR primer set common to PRSS3-SVs. (B) Expression level of PRSS3-SVs in HCC cell lines. Data from the DepMap (Table S1). (C) RT-qRCR of PRSS3 transcripts expressed in human fetal liver cell L02 and HCC cell lines. The relative expression level of each mRNA was normalized against $\beta$-Actin. (D) Comparison of the mRNA expression of PRSS3 and its transcript variants in 50 paired HCC and normal liver tissues (Table S2). The relative percentage of PRSS3 transcripts expressed in each paired sample (TPM scale) was visualized by $100 \%$ stacked bar graph. (E) The mRNA expression of PRSS3 transcripts in HCC tissues $(n=371)$ and normal liver tissues $(n=50)$ based on data from the FIREHOSE. The relative transcript level was presented as log2 $(T P M+1)$ scale. ${ }^{*} P<0.05$ by Wilcoxon rank sum test. 
a

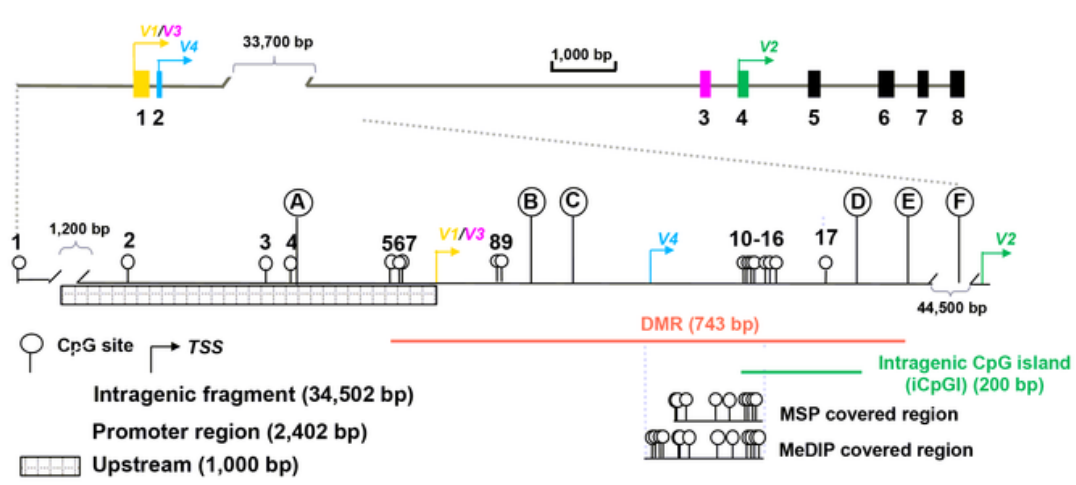

d

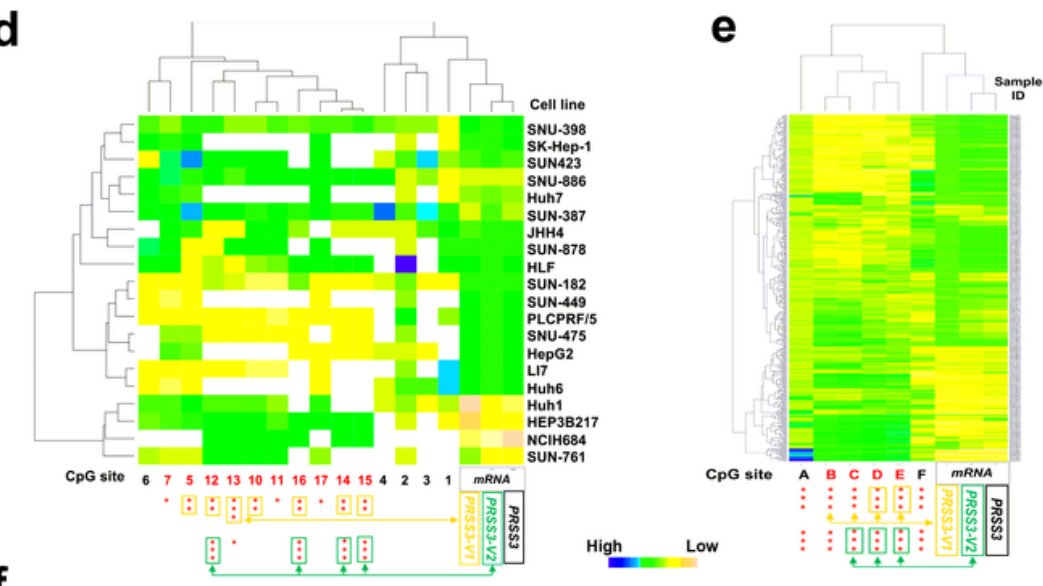

f
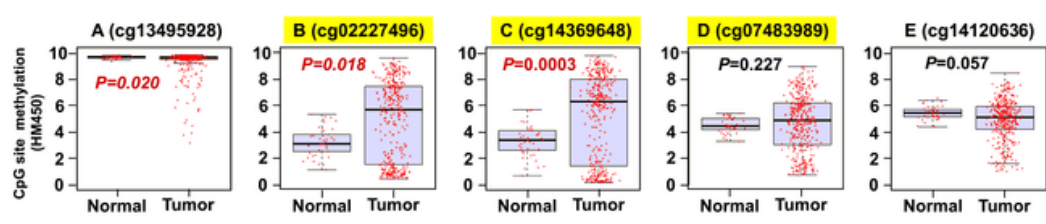

g
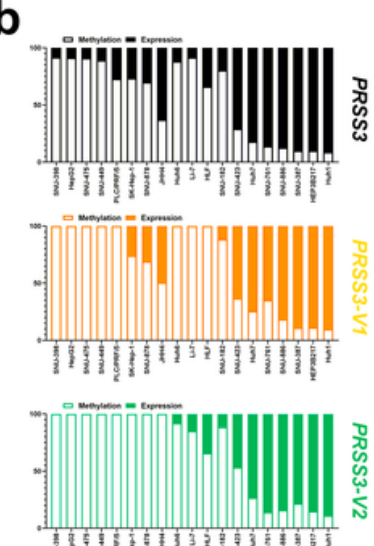

g

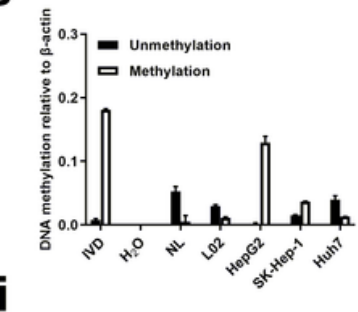

h
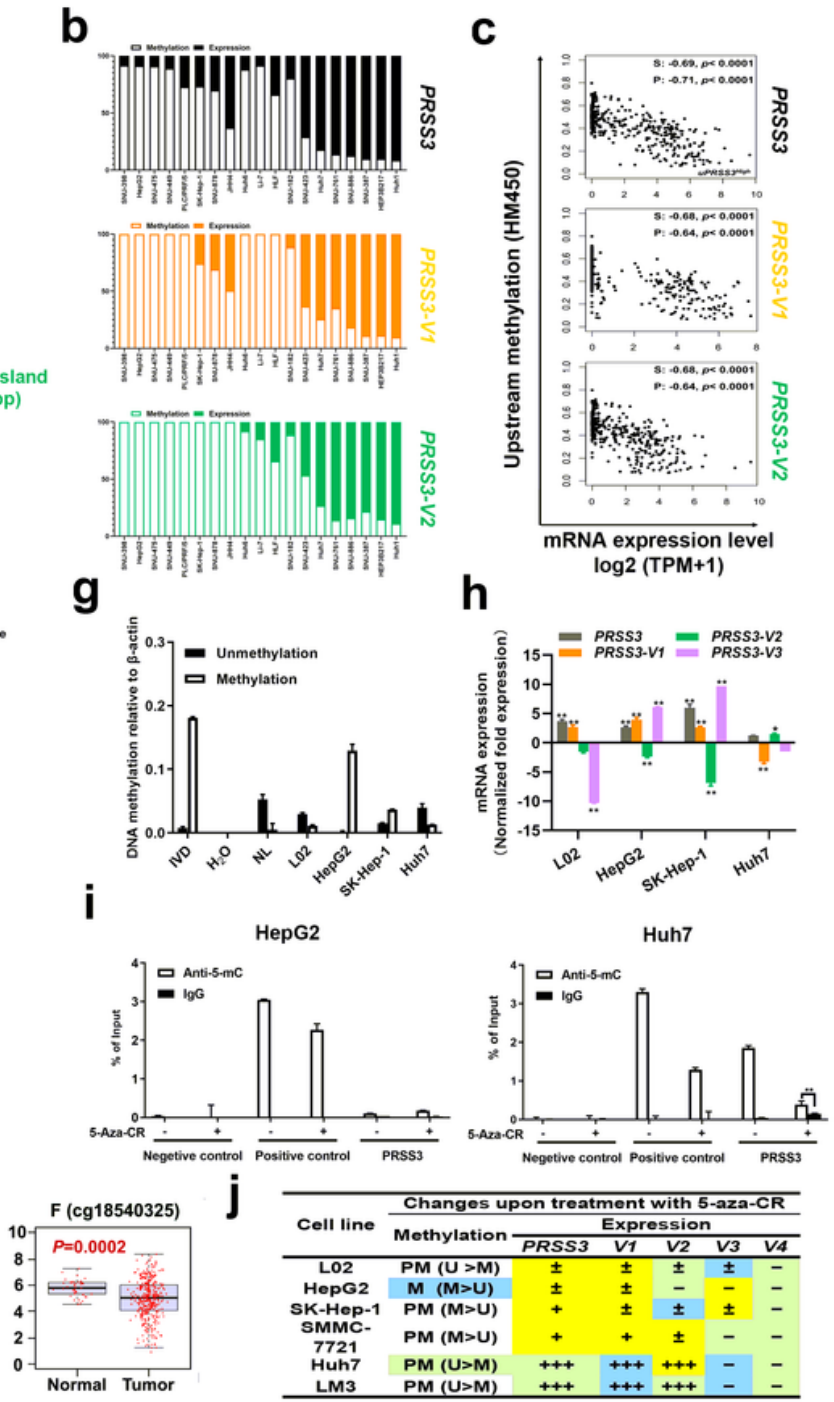

\section{Figure 3}

CpG methylation in regulation of the expression of PRSS3 transcripts in HCC. (A) Schematic of PRSS3 5'genomic region including extended promoter region, upstream and intragenic fragment. The promoter region (-1749 to $653 \mathrm{bp}$ ) shared by PRSS3-V1/3 contains 17 CpG sites (CpGs), including 5 CpGs (CpG site 2-7) in the $1 \mathrm{~kb}$ upstream fragment (-1000 bp from the TSS of PRSS3-V1/3). The extensive fragment includes 6 CpGs (defined as A, B, C, D, E and F) scattering around a broad genomic region about $34.5 \mathrm{~kb}$ scale from -170 down to 34,654 bp of the PRSS3-V1/3 TSS but still -10,643 bp away to the TSS of PRSS3-V2. The genomic position of each CpG site is shown relatively to the TSS of PRSS3-V1/3 (Table S4). Primer-covered regions for MSP-qPCR and MeDIP-qPCR are shown. (B) $1 \mathrm{~kb}$ upstream methylation normalized as percentage relative to PRSS3 expression in HCC cell lines visualized by $100 \%$ stacked bar graph. (C) Spearman and Pearson correlation analysis of $1 \mathrm{~kb}$ upstream methylation associated with PRSS3 expression in human primary liver tumor samples $(n=371)$. (D) and (E) Clustered heatmap of correlation between $\mathrm{CpG}$ site methylation and PRSS3 transcripts expression. Data was visualized by using correlation as distance function for heatmap cluster analysis of the promoter $\mathrm{CpG}$ methylation in $20 \mathrm{HCC}$ cell lines (D) and the intragenic 6 CpGs methylation in HCC tissue specimens ( $n=414)(E)$. In the 
heatmap blue color indicates low, green intermediate and yellow high DNA methylation or mRNA values. Rows: CpGs along correlatively with mRNA expression level of PRSS3 transcripts which were renormalized with mean $=0$, standard deviation $=1$ in accordance with DNA methylation values. Columns: HCC cell lines or tissue specimens. The statistical significance of correlation coefficients between CpG sites (red) and mRNA expression of PRSS3 transcripts were shown at the bottoms. * $\mathrm{P}<$ $0.05, * \star P<0.01, * \star * P<0.001$ (Figure S3, S4 and Table S5). (F) Association analysis of CpG Site methylation with PRSS3-SVs expression in $414 \mathrm{HCC}$ tissue specimens compared with 41 normal controls (Wilcoxon rank sum test). (G) MS-qPCR of PRSS3 methylation in HCC cell lines and L02 cells. In vitro methylated DNA (IVD) and normal human peripheral lymphocyte DNA (NL) served as positive and negative methylation controls, respectively. $(\mathrm{H})$ RT-qPCR of expression of PRSS3 transcripts in HCC cell lines and L02 cells upon treatment with epigenetic reagents, 5-aza-2'-deoxycytidine (5-Aza-CR: $5 \mu \mathrm{M}, 96$ h). ${ }^{*} P<0.05$, ${ }^{*} P<0.01$ by student's $t$ test. (I) MeDIP-qPCR to analyze 5 -mC-enriched genomic fragments associated with the intragenic $\mathrm{CpG}$ island (iCpGI) in HCC cell lines and L02 cells after 5-Aza-CR treatment. ** $P<0.01$ by student's $t$ test. $(J)$ In the summary table, the differential expression changes of PRSS 3 transcripts responding to treatment wirh 5-aza-CR was visualized with symbols and colors. The iCpGI methylation was defined as partial methylation (PM) or methylation (M) based on the MSP results. PRSS3 expression: "-",<0.001\%; " \pm ", 0.001-0.05\%; "+", > 0.05\%; "+++", > 1\%. The fold changes upon 5-aza$\mathrm{CR}$ treatment were shown in color: yellow, upregulation; green, downregulation; blue, no change. 

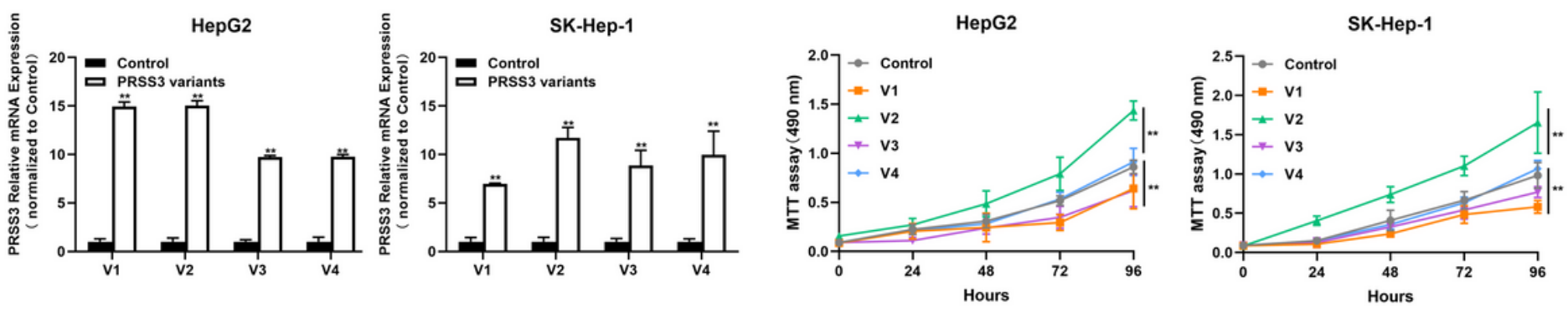

C
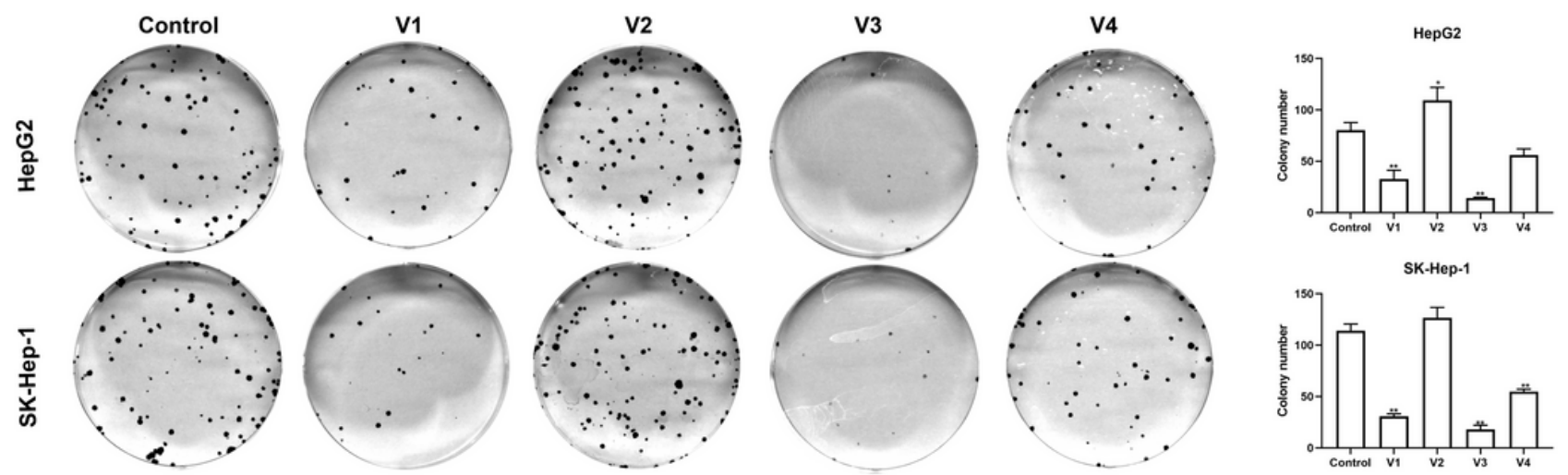

d
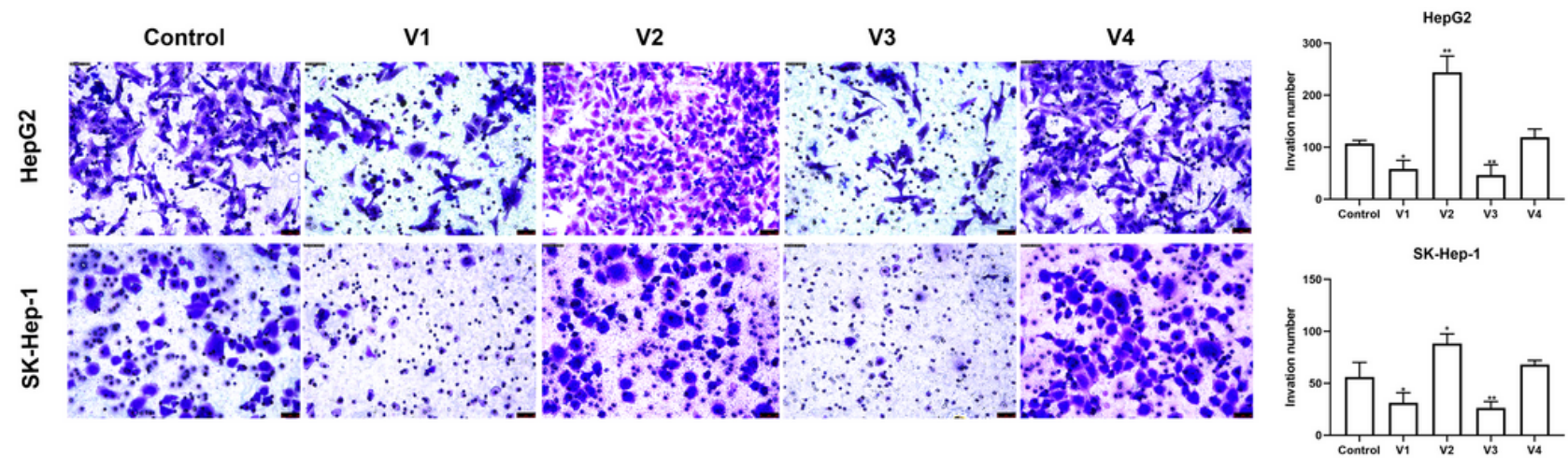

\section{Figure 4}

Effects of ectopically expressed PRSS3 transcripts on HCC cell malignancy. The PRSS3 splicing variants were separately transfected into HepG2 and SK-Hep-1 cells for establishment of the stable cell lines with individual overexpression of either PRSS3-V1 to -V4 (V1 to V4) or vector control (Control). (A) The mRNA expression levels of PRSS3 transcripts in the transfected cells were measured by RT-qPCR and quantified relative to the control cells (Student $t$ test). (B) Cell viability of HepG2 and SK-Hep-1 cells with ectopic expression of either PRSS3 transcript was detected by MTT assays ins comparison with the vector control (two tailed Student's t test). (C) Colony assays showing colony formation of HepG2 and SK-Hep-1 cells after overgrowing for 2 weeks. Representative images were presented on the left panel; quantitation of the colony numbers was shown on the right. (D) Transwell invasion assay assessing cell invasion capacity following transfection of PRSS3 transcripts. Left panel: representative image; Right panel: quantitation of the migrated cells. Scale bar: $50 \mu \mathrm{M}$. One-way ANOVA with Tukey's post hoc test was calculated for the transfected cells compared with the vector control in (C-D). ${ }^{*} P<0.05,{ }^{\star *} P<0.01$, versus 
control. Data are presented as mean \pm SD of a representative of three independent experiments done in triplicate.

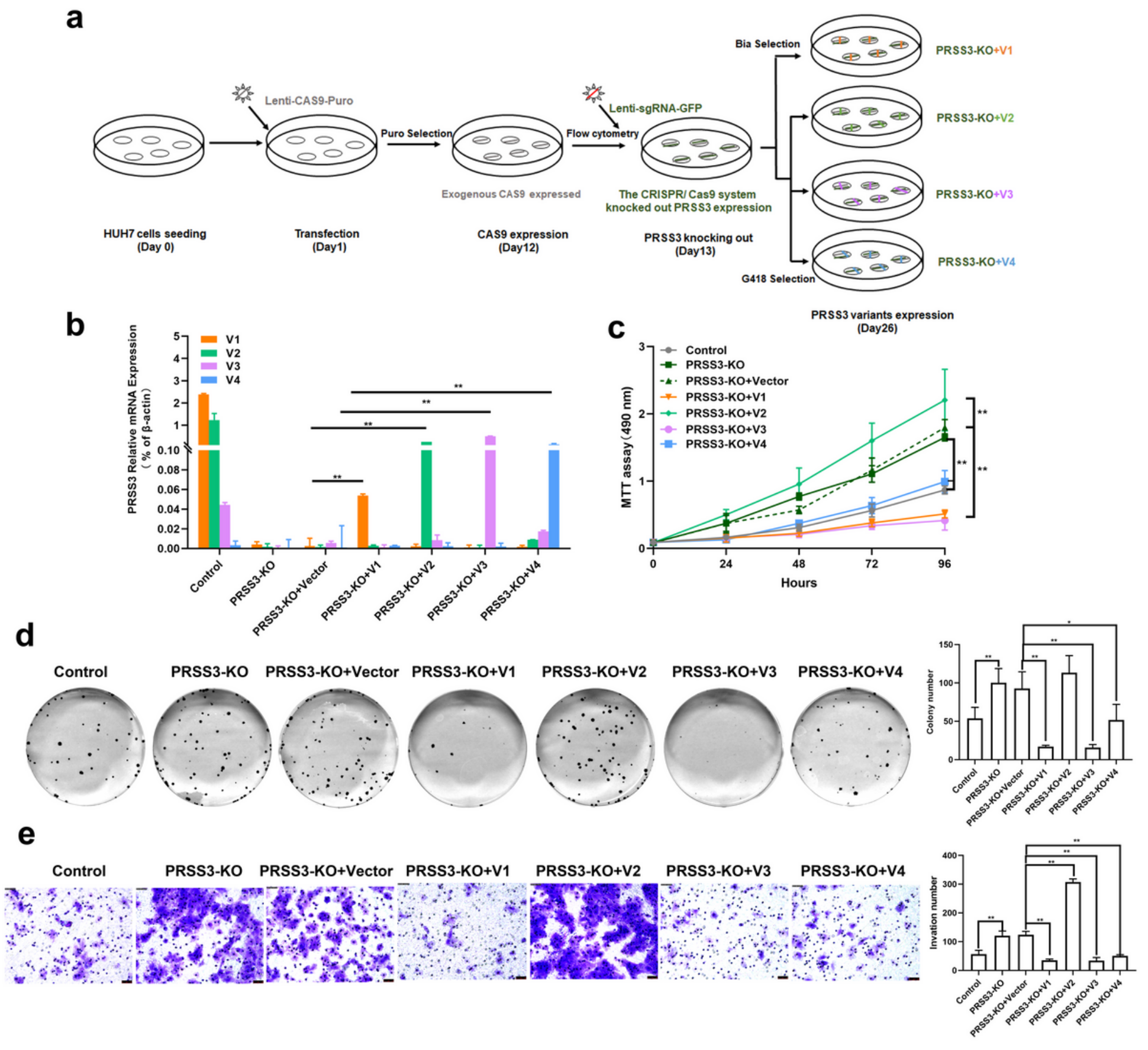

\section{Figure 5}

Functional divergence of PRSS3 transcript variants in a gain- and loss-of function cell model. (A) Schematic of workflow for construction of a cell model by endogenous knockout and then ectopic expression of PRSS3 transcript in Huh7 cells (PRSS3 KO+V cell model). Genomic deletion of PRSS3 transcripts by targeting common exon 5-8 region in PRSS3High Huh7 cells was performed using CRISPR/Cas9 system, followed by transfecting with PRSS3-V1 to -V4 constructs (PRSS3 KO+V1 to PRSS3KO+V4) or vector control (PRSS3KO+C), respectively. Puromycin (Puro) and Geneticin (G418) were used for selection of the transduced cells. (B) RT-qPCR analysis of PRSS3 mRNA expression in the 
transfected cells. The relative mRNA expression of PRSS3 transcripts normalized with $\beta$-actin (Student ttest). (C) MTT assays showed the viability of Huh7 cells (two tailed Student's t test). (D) Colony formation of Huh7 cells for 2 weeks. Left panel: representative image; Right panel: The colony numbers counted. (E) Transwell invasion assay assessing the invasion capacity of Huh7 cells upon the transfection. Left panel: representative images; Right panel: quantitation of the invaded cells. Scale bar: $50 \mu \mathrm{M}$. One-way ANOVA with Tukey's post hoc test was calculated for the transfected cells compared with the vector control in $(C-D) .{ }^{*} P<0.01 * * P<0.01$, versus control. Data is presented as mean $\pm S D$ of a representative of three independent experiments done in triplicate.

a

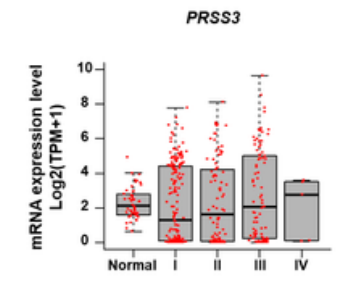

b

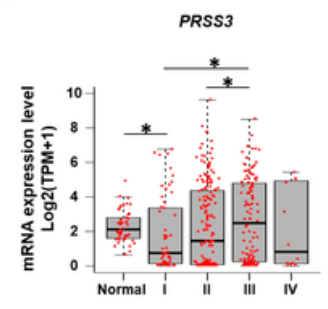

e

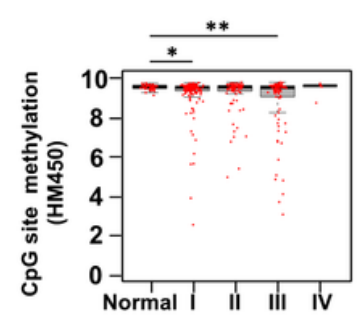

f

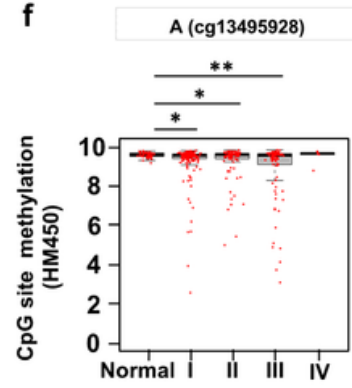

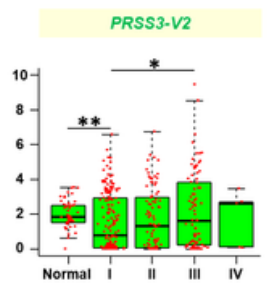

PRSS3-V2

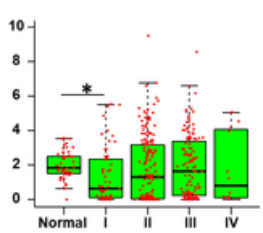

B (cg02227496)
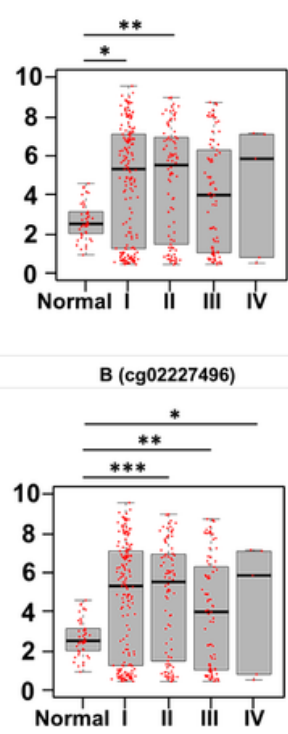

PRSS3-V1

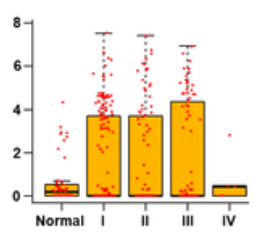

PRSS3.V1

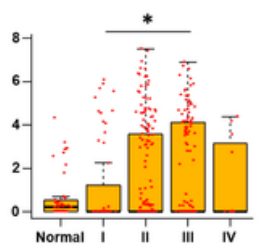

C (cg14369648)
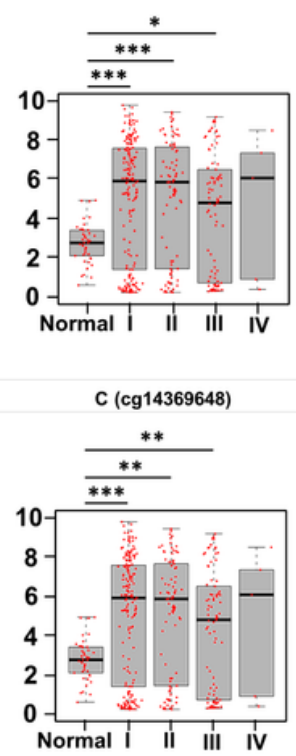

C
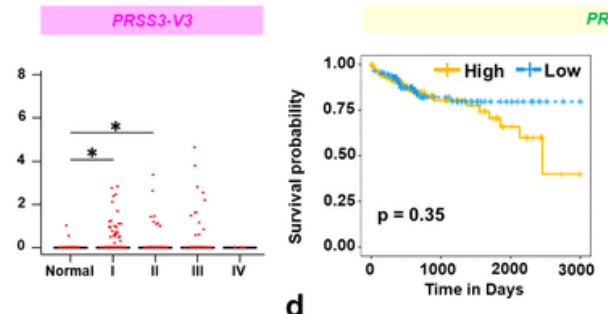

PRSS3-V2

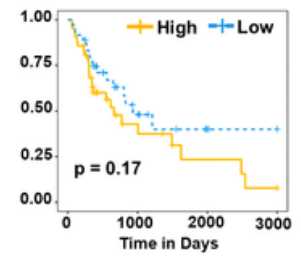

PRSS3-V2
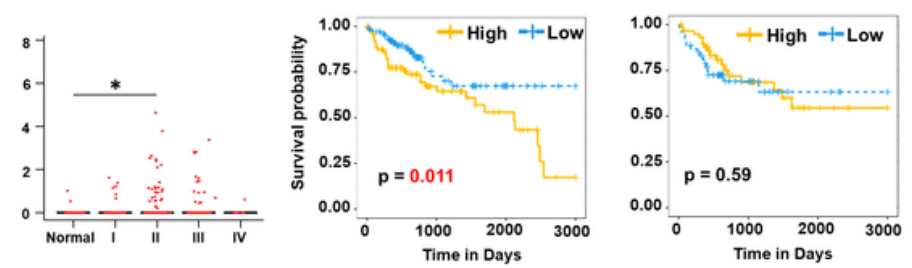

D (cg07483989)

$E(\operatorname{cg} 14120636)$
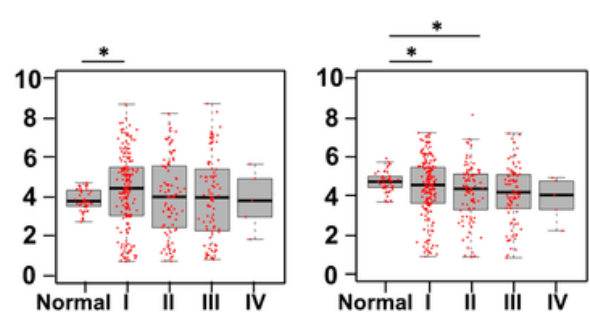

D (cg07483989)

E (cg14120636)
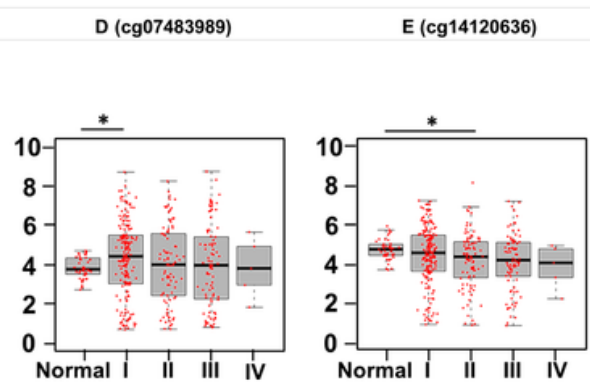
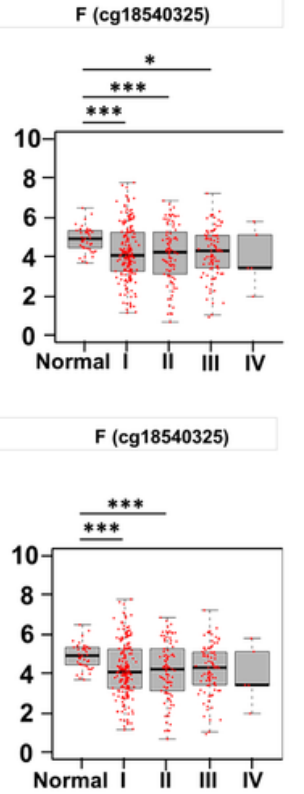

\section{Figure 6}

Clinical relevance in HCC patients in association with epigenetic alteration of PRSS3-SVs. (A and B) Boxand-Whisker plot with overlay of individual data points showing mRNA expression of PRSS3 transcripts in HCC tissues (Tumor $=371$ ) and normal controls (Normal $=50$ ), based on cancer stages (171 stage I, 86 stage II, 85 stage IIII, 5 stage IV) and tumor grades (55 grade I, 177 grade II, 122 grade III, 12 grade IV) (Table 1). (A) PRSS3; (B) PRSS3-SVs. (C and D) HCC patients were group into PRSS3-V2High and PRSS3- 
V2Lowgroups based on the mean value of each transcript in tumors (Table 1). The Kaplan-Meier method was used to determine the patient survival and log-rank (Mantel-Cox) test to compare survival rate. Results of HCC patients' survival curves from left to right panels: cancer stages I-II and III-IV (C), tumor grades I-II and tumor grades III-IV(D), respectively. The association of methylation of CpG site A-F within the extended fragment with different clinical stages $(E)$ and pathological grades $(F)$ in HCC tissue specimens $(n=414)$ in comparison with normal liver control tissues (Normal=41). The data was extracted from the FIREHOSE. Statistical significance was determined by Wilcoxon rank sum test. ${ }^{\mathrm{P}} \mathrm{P}<0.05$, $* \star \mathrm{P}<$ $0.01, * \star * P<0.001$.

\section{Image not available with this version}

\section{Figure 7}

A schematic model for epigenetic dysregulation of PPRSS3 transcripts functionally contributing to hepatocarcinogenesis and its biomarker potential. (A) Epigenetic silencing of PRSS3-SVs by site-specific CpG methylation in tumor of patients with early HCC, in which mPRSS3Low was a potential biomarker favorable for patients' survival. (B) Epigenetic disruption resulted in umPRSS3High in tumor of advanced HCC patients.

\section{Supplementary Files}

This is a list of supplementary files associated with this preprint. Click to download.

- SupFigandTableV1.docx

- Graphicalabstract.jpg 